\title{
4
}

\section{Rebuilding and Analysis of a SCIROCCO PWT Test on a Large TPS Demonstrator}

\author{
Sara Di Benedetto, Giuseppe C. Rufolo, Marco Marini and Eduardo Trifoni \\ CIRA, Italian Aerospace Research Centre \\ Italy
}

\section{Introduction}

In September 2007, a Plasma Wind Tunnel (PWT) Test was performed in the CIRA SCIROCCO facility on the FLPP Snecma Propulsion Solide (SPS) Thermal Protection System (TPS) demonstrator (Barreteau et al., 2008). Aim of the test was to verify, in a space qualifying environment, the behaviour of a large assembly constituted by Ceramic Matrix Composite (CMC) shingles, one curved and two flat panels, the same elements which will be part of the next ESA Intermediate Experimental Vehicle (IXV) thermal protection system. The focus of this chapter is the description of the CFD activities carried out in order to realize and support the plasma wind tunnel test, both in the phase of test definition and for the post test analysis.

During the pre-test CFD activity the test condition, previously defined by a simplified two dimensional methodology (Rufolo et al., 2008), has been verified by means of three dimensional simulations, and the final PWT test condition has been defined. Then, the posttest CFD rebuilding activity has allowed the analysis of results and the comparison with experimental measurements.

In addition, an assessment of the uncertainty level related to the satisfaction of the test requirements, in terms of heat flux and pressure to be realized over the test article, has been provided by analyzing the sources of error linked to both design and testing phases.

\section{Test requirements}

The test article is an assembly of CMC TPS elements: two flat panels located at 45 degrees with respect to the plasma flow and a curved panel which constitutes the model leading edge.

The test article configuration and its dimensions are represented in Fig. 1. Each portion of TPS to be tested (in white in figure) is separated by the other ones by a gap (1.5 $\mathrm{mm}$ in depth and $3 \mathrm{~mm}$ in width), in such a way to form a "T-gap" configuration.

The initial test design phase had been carried out in order to answer to the following customer requirements:

- cold-wall $\left(\mathrm{T}_{\mathrm{w}}=300 \mathrm{~K}\right)$, fully catalytic heat flux of $320 \mathrm{~kW} / \mathrm{m}^{2} \pm 10 \%$ at the beginning of the flat panels;

- constant wall pressure of 25 mbar $_{a}$ maintained during the test on the two flat panels surface. 
Moreover, the test article leading edge (curved panel) should not have been submitted, during the test, to a heat flux exceeding the value of $700 \mathrm{~kW} / \mathrm{m}^{2}$.

At the end of the test design activity (Rufolo et al., 2008), the PWT operating condition allowing the complete fulfilment of above requirements had resulted to be, in terms of facility reservoir conditions, $\mathrm{P}_{0}=5.2$ bar $_{\mathrm{a}}$ and $\mathrm{H}_{0}=16.7 \mathrm{MJ} / \mathrm{kg}$, realized with the SCIROCCO conical nozzle D, characterised by a $1.15 \mathrm{~m}$ nozzle exit diameter, and with the model stagnation point located $0.35 \mathrm{~m}$ downstream of the nozzle exit section.

The achievement of the desired operating condition $\left(\mathrm{P}_{0}, \mathrm{H}_{0}\right)$ in test chamber is assured by the measurements of stagnation heat flux and pressure on a water cooled copper probe. The stagnation values corresponding to the reservoir conditions above, and determined by CFD during the test design phase, were: $P_{s}=36.15$ mbar $_{a}$ and $Q_{s}=2070 \mathrm{~kW} / \mathrm{m}^{2}$.

A complete description of the SCIROCCO facility is given in the following section.

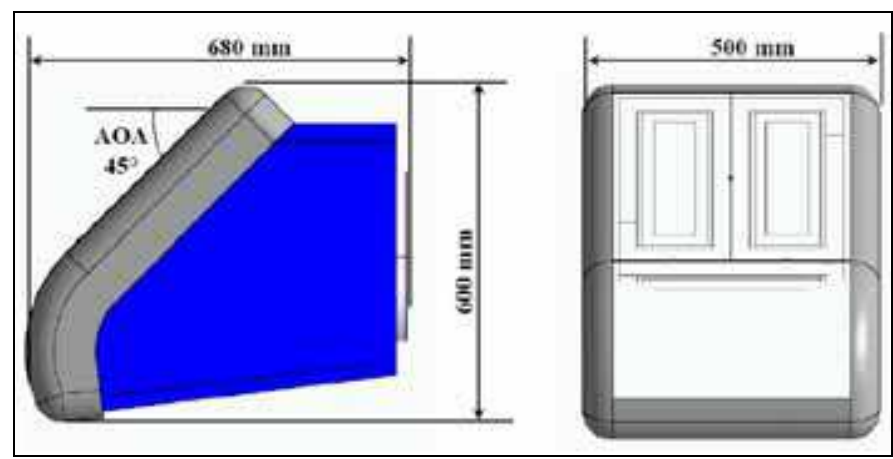

Fig. 1. Test article geometry

\section{SCIROCCO plasma wind tunnel}

The CIRA SCIROCCO Plasma Wind Tunnel (Marini et al., 2002 and De Filippis et al., 2003) is devoted to aerothermodynamic tests on components of aerospace vehicles; its primary mission is to simulate the thermo-fluid-dynamic conditions suffered by full scale Thermal Protection System (TPS) of space vehicles re-entering the Earth atmosphere.

SCIROCCO is a large size facility (see Fig.2), whose hypersonic jet impacts the test article with a diameter size up to $2 \mathrm{~m}$ and reaches Mach number values up to 11 . The jet is then collected by a long diffuser $(50 \mathrm{~m})$ and cooled by an heat exchanger. Seventy MW electrical power is used to heat the compressed air that expands along a converging-diverging conical nozzle. Four different nozzle exit diameters are available: 0.9, 1.15, 1.35 and $1.95 \mathrm{~m}$, respectively named $\mathrm{C}, \mathrm{D}, \mathrm{E}$ and $\mathrm{F}$.

The overall performance of SCIROCCO in terms of reservoir conditions is the following: total pressure $\left(\mathrm{P}_{0}\right)$ varies from 1 to 17 bar and total enthalpy $\left(\mathrm{H}_{0}\right)$ varies from 2.5 to 45 $\mathrm{MJ} / \mathrm{kg}$. Enthalpy values between 2.5 and $10 \mathrm{MJ} / \mathrm{kg}$ are obtained using a plenum chamber between the arc heater column exit and the nozzle inlet converging part, which allows transverse injection of high pressure ambient air to reduce the flow total enthalpy.

The energetic heart of the facility is the segmented constricted arc heater, a column with a maximum length of $5.5 \mathrm{~m}$ and a bore diameter of $0.11 \mathrm{~m}$. At the extremities of this column there are the cathode and the anode between which the electrical arc is generated. A power 


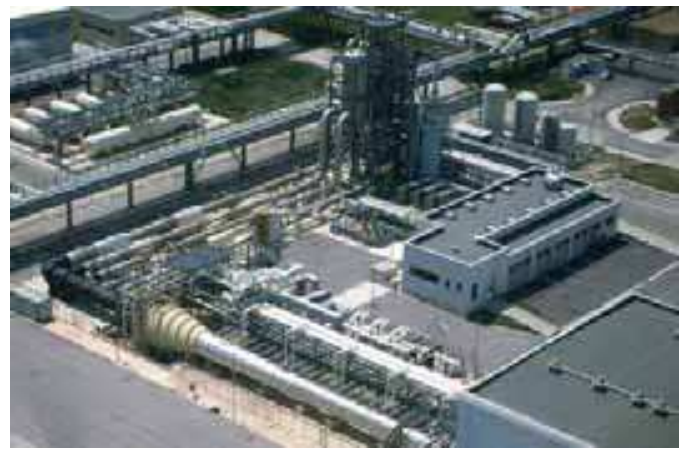

Fig. 2. SCIROCCO Plasma Wind Tunnel aerial view

supply feeds the electrical DC power to the electrodes for the discharge. A compressed air supply distributes dry compressed air to the various segments of the arc heater column, being able to supply a mass flow rate ranging from 0.1 to $3.5 \mathrm{~kg} / \mathrm{s}$, heated up to $10000 \mathrm{~K}$.

The last important subsystem of SCIROCCO is the vacuum system, which generates the vacuum conditions in test chamber required by each test. The system consists of ejectors that make use of high pressure water steam as motor fluid $\left(28.5\right.$ bar $_{\mathrm{g}}$ and $\left.250{ }^{\circ} \mathrm{C}\right)$.

Facility theoretical performance map in terms of reservoir conditions produced by the arc heater is shown in Fig. 3.

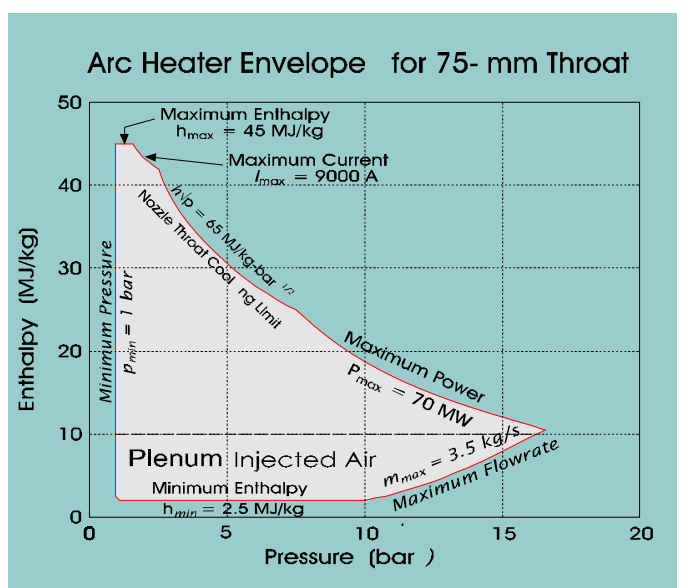

Fig. 3. Arc heater theoretical performance map

The achievement of the desired operating conditions $\left(\mathrm{P}_{0}, \mathrm{H}_{0}\right)$ in test chamber before the insertion of the model is assured by the measurements of stagnation pressure $\left(\mathrm{P}_{\mathrm{s}}\right)$ and stagnation heat flux $\left(Q_{\mathrm{s}}\right)$ radial profiles on a $100 \mathrm{~mm}$-diameter hemi-spherical calibration probe, made of copper and water cooled, at a section $0.375 \mathrm{~m}$ downstream of the conical nozzle exit section, by means of high precision pressure transducers and Gardon-Gage heat flux sensors, respectively. Facility regulations (mass flow, current) are tuned in order to measure on the calibration probe a certain couple of values $\left(P_{s}, Q_{s}\right)$ which corresponds to the desired set point in terms of the couple $\left(\mathrm{P}_{0}, \mathrm{H}_{0}\right)$. 


\section{Numerical methodology}

\subsection{Numerical tool}

All the three-dimensional numerical computations presented in this chapter have been performed by using the CIRA CFD code H3NS.

H3NS is a structured multi-block finite volume solver that allows for the treatment of a wide range of compressible fluid dynamic problems, and has been widely validated in the past (Ranuzzi \& Borreca, 2006), (Di Clemente, 2008).

It solves the full Navier-Stokes equations for a real gas in thermal and chemical nonequilibrium conditions. The governing equations, written in conservation form, are discretized by using a finite volume technique with a centred formulation; the inviscid fluxes are computed by means of a Flux Difference Splitting (FDS) Riemann solver, with a second order ENO reconstruction of interface values, whereas viscous fluxes are calculated by central differencing, i.e. computing the gradients of flow variables at cell interfaces by means of the Gauss theorem. Time integration is performed with an explicit Euler forward algorithm and a Local Time Stepping formulation, coupled with a point-implicit evaluation of chemistry and vibrational source terms.

In the case of thermo-chemical non equilibrium flows the fluid is treated as a mixture of perfect gases. The chemical model for air is due to Park (Park, 1989) and it is characterized by 17 reactions between the five species $\left(\mathrm{O}, \mathrm{N}, \mathrm{NO}, \mathrm{O}_{2}, \mathrm{~N}_{2}\right)$, neglecting the presence of inert gas or water in the air. The energy exchange between vibrational and translational modes is modeled with the classical Landau-Teller non-equilibrium equation, with relaxation times taken from the Millikan-White theory (Millikan \& White, 1963) modified by Park (Park \& Lee, 1993). The viscosity of the single species is evaluated by a fit of collision integrals calculated by Yun and Mason (Yun \& Mason, 1962); the thermal conductivity is calculated by means of the Eucken law; the viscosity and thermal conductivity of the gas mixture are then calculated with the semi-empirical Wilke's formulae. The diffusion of the multi-component gas is computed through a sum rule of the binary diffusivities of each couple of species (Kee et al., 1983). Transport coefficients, assuming ideal gas, are derived from Sutherland's law.

Several models for the treatment of finite rate catalysis are implemented both considering a constant recombination coefficient and ad hoc developed model for TPS materials (e.g. Di Benedetto \& Bruno, 2010).

\subsection{Three-dimensional computational grid}

The three-dimensional computational grid around the test article has been generated by means of the commercial software ANSYS ICEMCFD ${ }^{\circledR}$.

Grid, composed of hexahedral elements, has been generated for half model using a multiblock approach, and has been stretched normally to wall surfaces in order to properly predict the different boundary layers developing around the geometric configuration. The topology of the grid has been created in order to accurately define all the geometric details of test article and obtained by using a certain number of O-grids (Fig. 4) for the block decomposition.

The computational grid on the full test article is shown in Fig. 5, while an enlargement of top frame is depicted in Fig. 6.

Within the main O-grid containing the body, two O-grids have been generated around the curved and flat panel, respectively; in this way it is possible to keep down the overall number of grid points still preserving a good discretization of the gaps (see Fig. 7). 
It must be underlined that these gaps have been modelled with sharp edges (a measure of local curvature radii was not available), therefore results in terms of heat flux peaks are conservative.

Moreover, the bow shock wave surface has been properly fitted. In order to minimize the numerical instabilities that propagate from the shock wave towards the stagnation region (the "carbuncle" phenomenon), it is important to align as much as possible the grid lines to the shock.

Grid characteristics are listed in Tab. 1, $\Delta n_{\min }$ being the minimum spacing normal to the wall at the stagnation point and $A R$ the corresponding aspect ratio. Three grid levels have been adopted, in order to assure grid convergence of results, as it will be shown in Section 5.2.

\begin{tabular}{|c|c|c|c|}
\hline Grid Level & Cells & $\Delta n_{\min }$ & $A R$ \\
\hline coarse & 32468 & $7 \cdot 10^{-6} \mathrm{~m}$ & 2500 \\
medium & 259744 & $2 \cdot 10^{-6} \mathrm{~m}$ & 4050 \\
fine & 2077952 & $1 \cdot 10^{-6} \mathrm{~m}$ & 4410 \\
\hline
\end{tabular}

Table 1. Computational grid characteristics

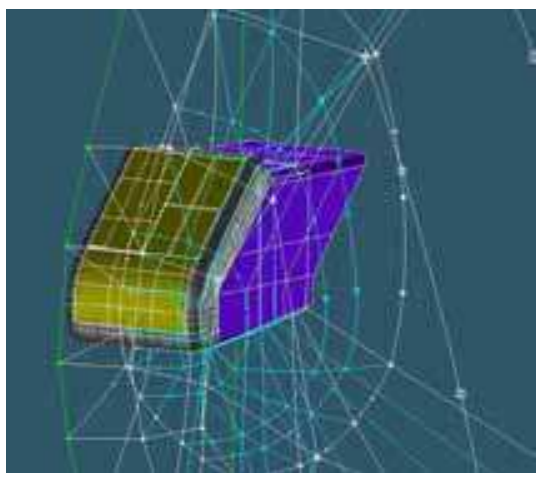

Fig. 4. Block decomposition

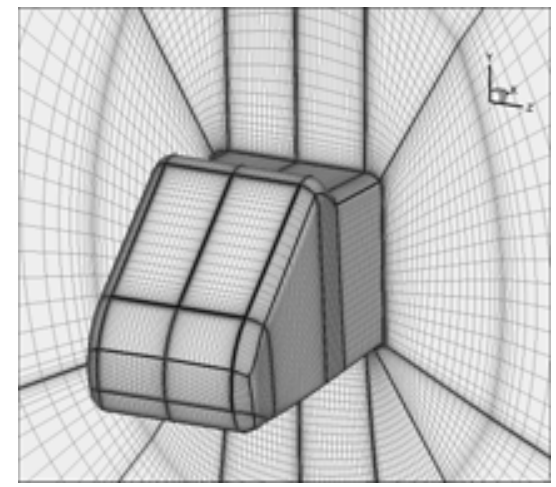

Fig. 5. Three dimensional computational grid 


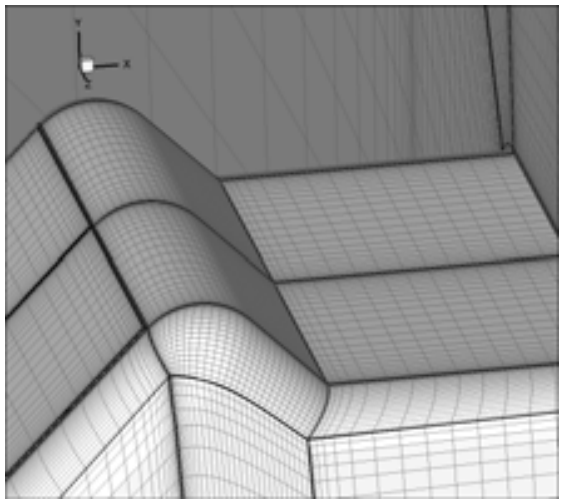

Fig. 6. Detail of the top frame
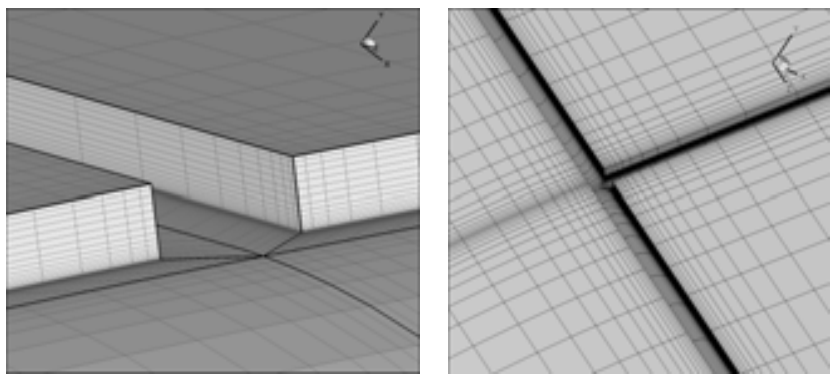

Fig. 7. Gap between panels and frame (left) and T gap (right)

\section{Pre Test CFD activity}

In this section CFD three dimensional results of the flow field computed around the test article are shown and deeply analyzed for the PWT condition selected during the test design phase (Rufolo et al., 2008), i.e. $P_{s}=36.15$ mbar $_{a}$ and $Q_{s}=2070 \mathrm{~kW} / \mathrm{m}^{2}$. Subsequently, grid convergence of results will be shown in Section 5.2, and an assessment of the uncertainty level linked to both design and testing phases will be presented in Section 5.3.

\subsection{Three-dimensional results and test requirements verification}

Three-dimensional computations on the full test article configuration have been performed with the aim at verifying the test requirements fulfilment with the PWT condition defined. Moreover, information about flow features (presence of vortex structures, separation and reattachment lines, overheatings induced by the gaps, etc.) and spanwise effects will be given in the following, in order to exactly account for the overheatings predicted on the lateral parts of the CMC panels.

The computation has been performed for half model and in the hypothesis of cold $\left(\mathrm{T}_{\mathrm{w}}=300\right.$ $\mathrm{K})$ and fully catalytic wall, as requested by SPS at the end of the test design phase.

Mach number and pressure contour maps are shown in Fig. 8. The shape of the bow shock around the model is clearly predicted as well as the stagnation pressure region (on the curved panel), the constant pressure region on the model flat panel and the strong 
expansions occurring in correspondence of the roundings, either on the top frame either on the lateral fairings.
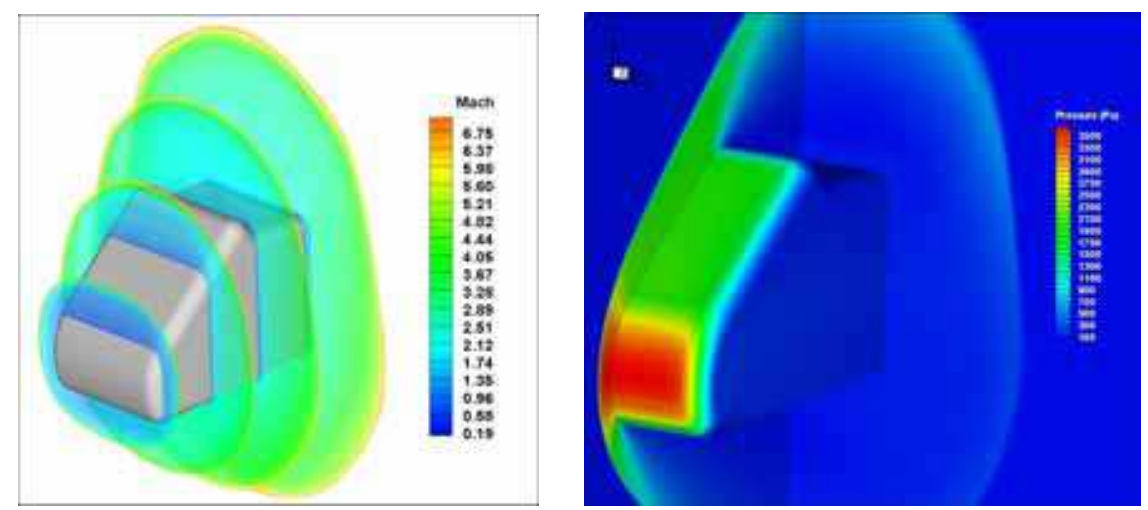

Fig. 8. Mach number around the model (left) and pressure contour map (right)

The first verification has concerned with the possibility of wind tunnel blockage occurrence due to the large size and bluntness of the FLPP-SPS model. As shown in Fig. 9, where the computed two-dimensional and three-dimensional bow shock shapes in the model centre plane are reported, evident finite span effects are present for this test article which make the bow shock closer to the TPS demonstrator with respect to the design solution.

The reason is the spanwise flow induced by the strong transversal pressure gradient, due to the $45 \mathrm{deg}$ inclination of the panels with respect to the free stream.

Fig. 10 shows the model with its bow shock wave inside the test chamber and in front of the diffuser entrance, at the position of $0.35 \mathrm{~m}$ downstream of the nozzle exit section. It is evident that the bow shock wave is fully swallowed by the diffuser pick-up.

This occurrence constitutes a necessary condition to be verified in order to exclude the risk of wind tunnel blockage.

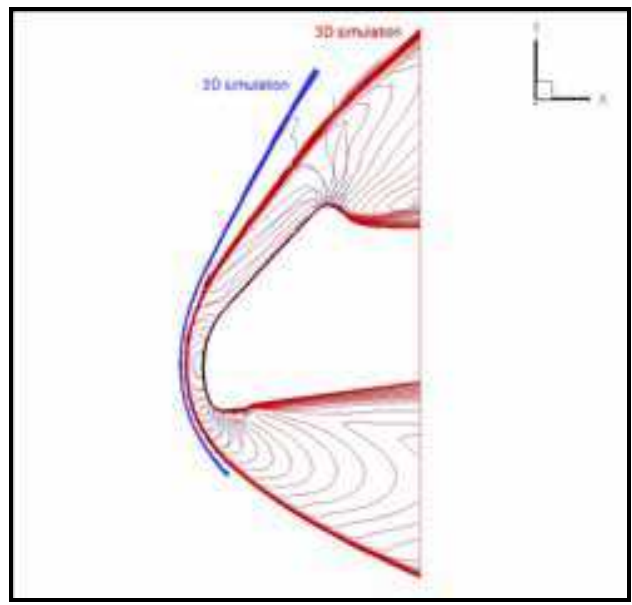

Fig. 9. Bow shock in the symmetry plane 


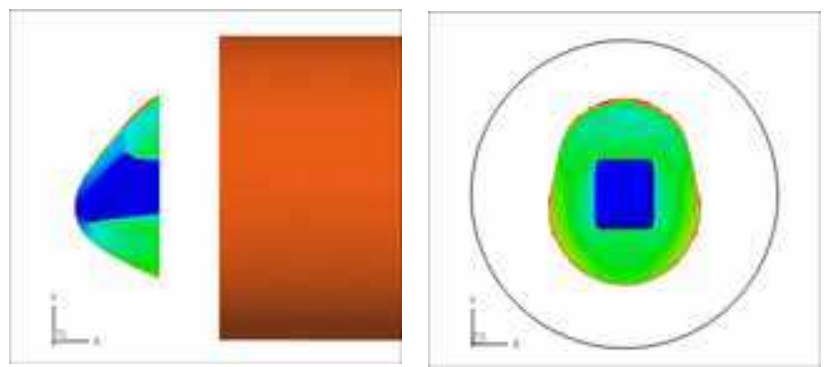

Fig. 10. Side (left) and front (right) view of the model with its bow shock ahead the diffuser entrance

Fig. 11 shows the heat flux distribution predicted on the full model together with the skinfriction lines pattern (the solution on half model has been mirrored with respect to the symmetry plane).

The stagnation line on the curved panel and the local maximum values of heat flux (less than $1 \mathrm{MW} / \mathrm{m}^{2}$ ) at the roundings of the lateral fairings of the curved panel can be clearly observed in the same figure, as well as the strong three-dimensionality of the flow over this model, that also affects the region close to the symmetry plane, where test requirements have been defined and matched in the test design activity (Rufolo et al., 2008).

An enlargement of the model top frame is reported in Fig. 12, where the skin friction lines are coloured depending on the local shear stress value. The local maxima of shear stress are predicted at the shoulder of the top frame and at the roundings of the lateral fairings, as expected, due to the turning of the flow with associated boundary layer thinning.

A large separated area (with negative values of shear stress) is clearly visible on the top frame caused by the local shock wave boundary layer interaction, with a nearly straight separation line and a highly distorted attachment line; the extent of the separated flow area increases at the extremities due to the inlet of the flow turning around the model.

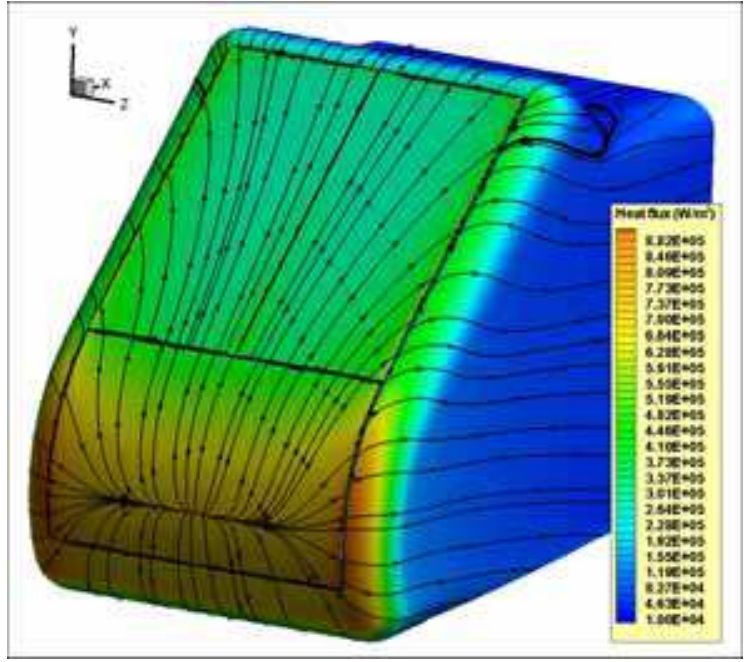

Fig. 11. Heat flux contour map with skin-friction lines 


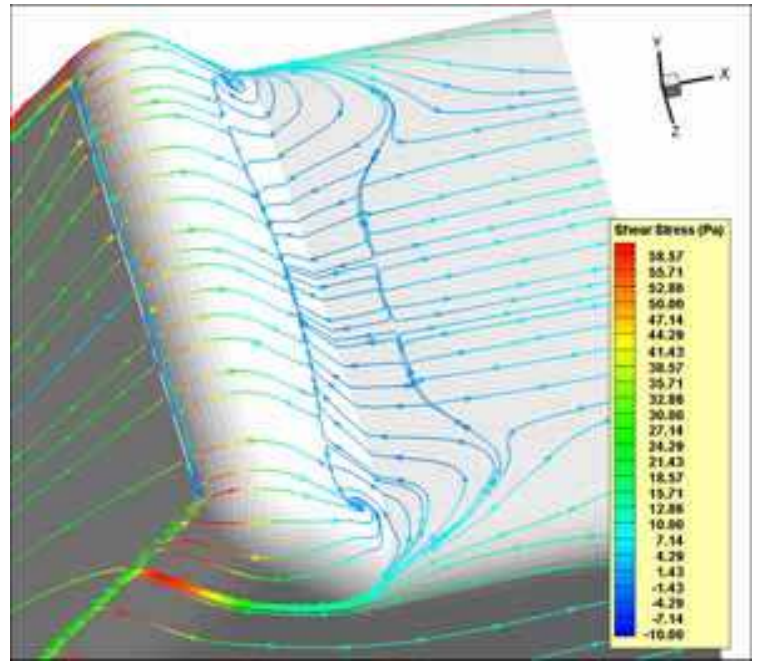

Fig. 12. Enlargement of the model top frame; skin-friction lines coloured by the shear stress

The lower frame heat flux contour map and the related skin friction lines are reported in Fig. 13, showing a nearly two-dimensional recirculation induced by the presence of the step, with maximum heat flux values ranging from $45 \mathrm{~kW} / \mathrm{m}^{2}$ in the central lower frame area to $90 \mathrm{~kW} / \mathrm{m}^{2}$ at the edges, where flow recirculation disappears due to the particular transversal shape of the model bottom part.

The flow inside the longitudinal gap existing between the two flat panels, and inside the transversal gap between the full span curved panel and the two flat panels (T-gap structure), is described in detail from Fig. 14 to Fig. 16. A flow recirculation is predicted inside the longitudinal gap (see Fig. 14), with a complex vortex pattern in the "T-gap" region (see Fig. 15). The vortex flow inside the transversal gap is characterized by a strong spanwise velocity component, that increases moving towards the edge, a inner vortex at the base of the panel and an attachment line at the front edge of the panel, where very high heat flux values $\left(\sim 1 \mathrm{MW} / \mathrm{m}^{2}\right)$ are predicted in a very small region.

Fig. 16 describes the exit of the transversal gap flow into the external flow developing on the lateral fairing. The interaction of the two streams causes a rapid turning of the transversal gap flow with the formation of a local saddle point. It should be also underlined the presence of a inner vortex developing parallel to the junction between the flat panel and the lateral fairing, and the presence of an attachment line (the same already seen in Fig. 15) at the front edge of the flat panel, which corresponds to a region of high heat flux, with a maximum in the top corner of about $1.6 \mathrm{MW} / \mathrm{m}^{2}$ but localized in a very small region $(0.0002$ $\mathrm{m}$ depth).

In order to verify test requirements in terms of heat flux and pressure at the beginning of the flat panel, and to properly evaluate spanwise and viscous effects, the longitudinal and transversal distributions along the slices indicated in Fig. 17 have been analyzed.

Results in terms of heat flux are reported in Fig. 18 and Fig. 19, showing transversal and longitudinal distributions, respectively, these latter ones compared to the two-dimensional results of test design activity (Rufolo et al., 2008). 


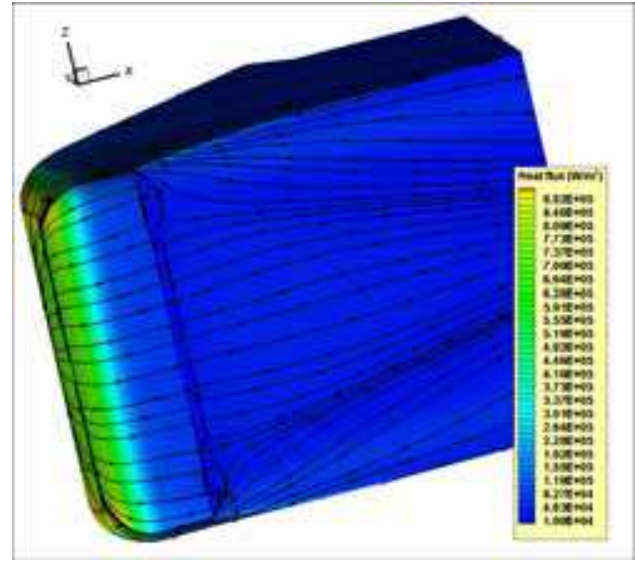

Fig. 13. Heat flux contour map with skin-friction lines; model bottom frame

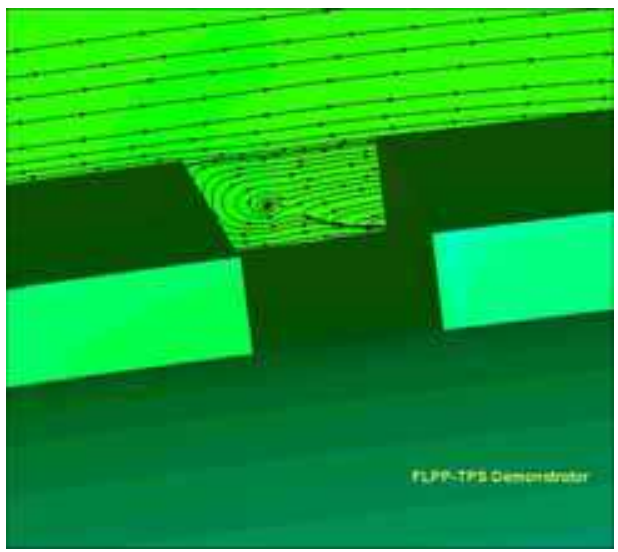

Fig. 14. Re-circulating region; longitudinal gap

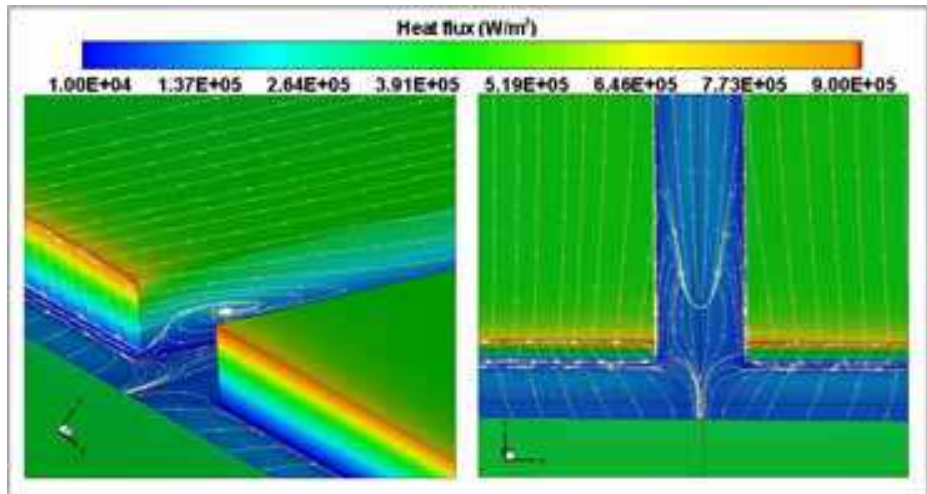

Fig. 15. T-gap; heat flux contour map with skin-friction lines 


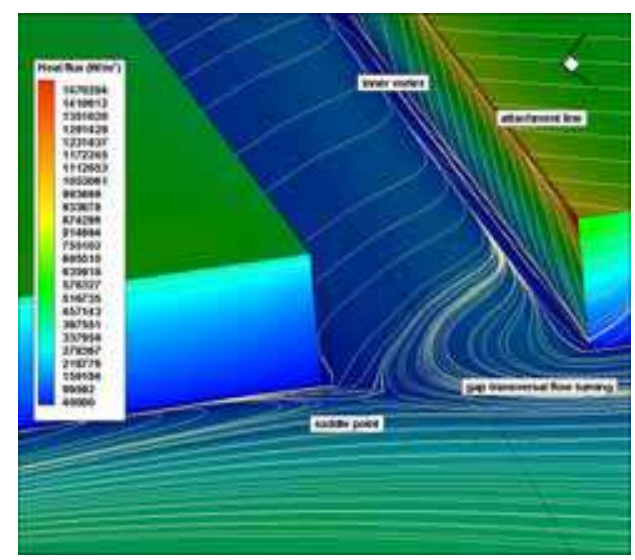

Fig. 16. Exit of transversal gap flow. Heat flux contour map and skin-friction lines

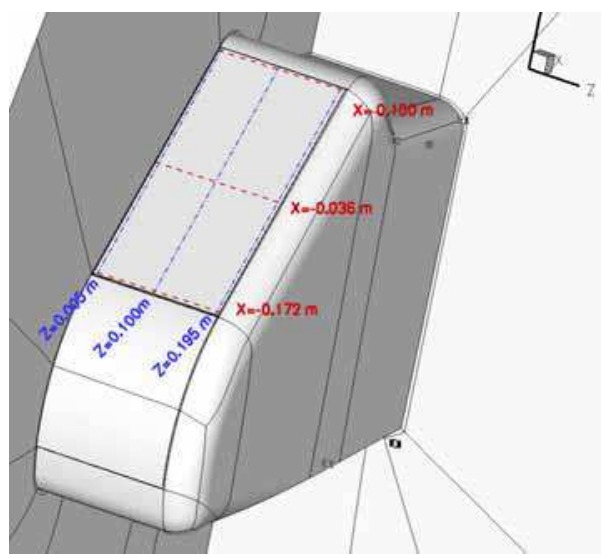

Fig. 17. Longitudinal and transversal slices

The increase of heat flux predicted on the flat panel is due either to spanwise effects either to the presence of gaps (longitudinal and transversal) and steps (lateral side), as clearly shown in Fig. 18. At the flat panel leading edge three-dimensional CFD simulation yields a $28 \%$ increase $\left(450 \mathrm{~kW} / \mathrm{m}^{2}\right)$ of predicted heat flux, both $5 \mathrm{~mm}$ from the centreplane $(\mathrm{Z}=0.005 \mathrm{~m})$ and $5 \mathrm{~mm}$ from the lateral edge $(Z=0.195 \mathrm{~m})$, and it is nearly $350 \mathrm{~kW} / \mathrm{m}^{2}$ in-between. Downstream along the panel the predicted heat flux is closer to the test requirement, while localized high heat flux peaks are present in correspondence of gaps and steps.

Transversal and longitudinal wall pressure distributions are shown in Fig. 20 and Fig. 21, respectively. Pressure is not affected by spanwise effects from the qualitative point of view (the transversal distributions remain two-dimensional for most of the half panel span), but a quantitative reduction of $17 \%$ of maximum pressure on the flat panel centreplane is predicted (2070 $\mathrm{Pa}$ instead of $2500 \mathrm{~Pa})$. 


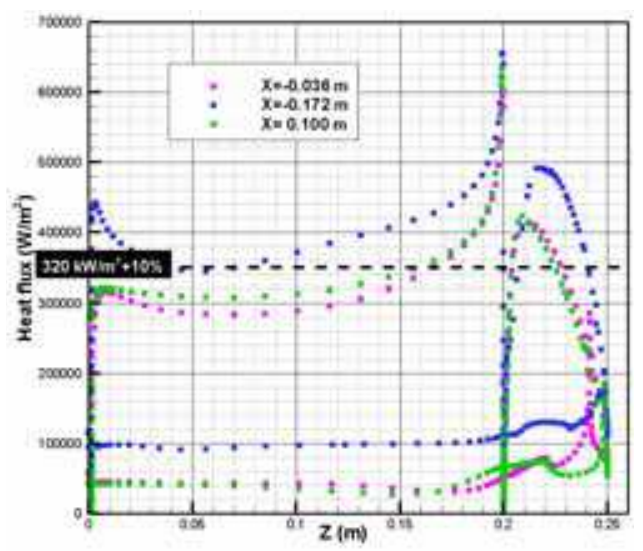

Fig. 18. Transversal heat flux distributions

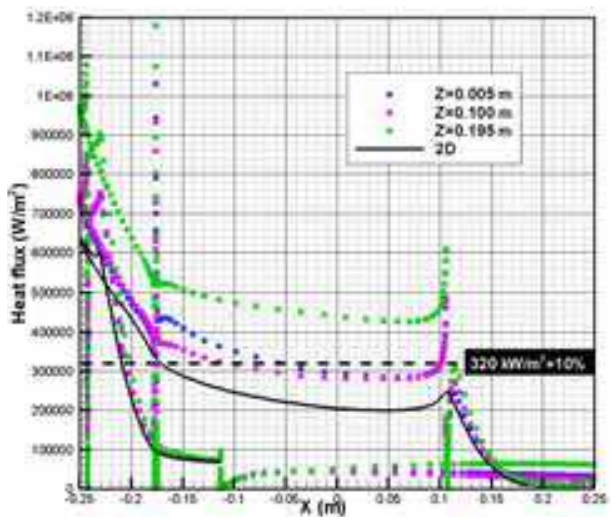

Fig. 19. Longitudinal heat flux distributions; comparison with 2D distribution

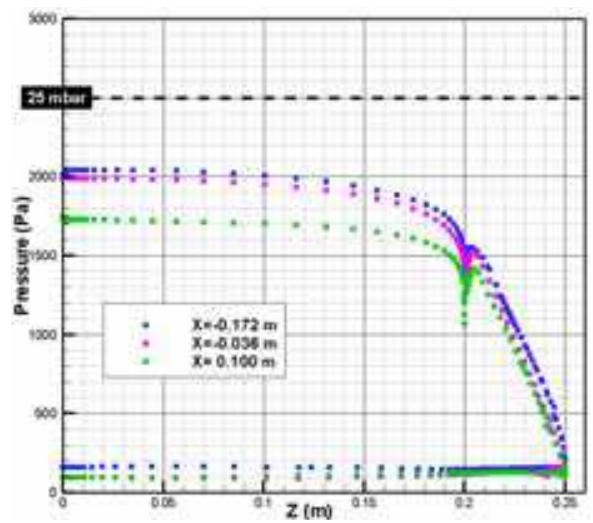

Fig. 20. Transversal wall pressure distributions 


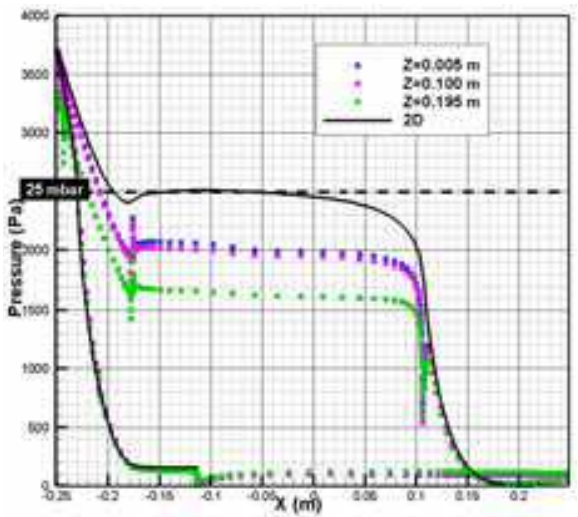

Fig. 21. Longitudinal wall pressure distributions; comparison with 2D distribution

\subsection{Grid convergence of results}

Grid convergence study is the most common and reliable technique for the quantification of numerical uncertainty (Roache, 1998) related to spatial discretization. It has been carried out for the three-dimensional pre-test computation by using the different grid levels indicated in Tab. 1.

Temporal convergence of the solutions has been obtained on all the grid levels.

Grid convergence of results has been evaluated in correspondence of the same points used in the design phase for monitoring the test requirements matching, i.e. the beginning of flat panel for the heat flux and the point of maximum value for the pressure on the flat panel, both taken at the centreline. In the three-dimensional case, these control points have been selected in the spanwise direction in order to be close to the symmetry plane, but sufficiently far from the region affected by the presence of the longitudinal gap; their coordinates are reported in Tab. 2.

$\mathrm{Q}^{*}$ and $\mathrm{P}^{*}$ indicate the values of heat flux and pressure in the selected points.

\begin{tabular}{|c|c|}
\hline \multicolumn{2}{|c|}{$\mathrm{z}=0.07 \mathrm{~m}$} \\
\hline $\mathbf{x}$ & $\mathbf{x}$ \\
(for Q evaluation) & (for P evaluation) \\
$\mathbf{- 0 . 1 7 2 ~} \mathbf{~ m}$ & $\mathbf{- 0 . 1 5 6 ~} \mathbf{~ m}$ \\
\hline
\end{tabular}

Table 2. Coordinates of the points selected for the grid convergence study

\begin{tabular}{|c|c|c|c|c|}
\hline GRID & $\mathbf{N}$ & $\mathbf{N}^{-1 / 3}$ & $\mathbf{Q}^{*}\left(\mathbf{W} / \mathbf{m}^{2}\right)$ & $\mathbf{P}^{\star}(\mathbf{P a})$ \\
\hline coarse & 32468 & 0.0313 & 132675.69 & 1959.30 \\
medium & 259744 & 0.0157 & 335118.84 & 2024.86 \\
fine & 2077952 & 0.0078 & 349148.53 & 2044.60 \\
Rich.Extrap. & inf. & $\mathbf{0}$ & $\mathbf{3 5 3 8 2 5 . 0 9}$ & $\mathbf{2 0 5 1 . 1 9}$ \\
\hline
\end{tabular}

Table 3. $\mathrm{Q}^{*}$ and $\mathrm{P}^{*}$ values at the selected points for the three grid levels and Richardson Extrapolation 
The computed $\mathrm{Q}^{*}$ and $\mathrm{P}^{*}$ values are reported in Tab. 3 for the three grid levels, together with the Richardson Extrapolation value. This latter is an estimation of the "continuum value" (i.e., the value at zero grid spacing), obtained from a series of discrete values, and it is defined in the following way:

$$
f_{h=0} \cong f_{1}+\frac{f_{1}-f_{2}}{r^{p}-1}
$$

where: $f_{h=0}$ is the value at zero grid spacing; $f_{1}$ and $f_{2}$ are the values computed on two grids, $f_{1}$ being the finer one; $p$ is the order of the solution ( $p=2$ for this case); $r$ is the grid refinement ratio:

$$
r=\sqrt[3]{\frac{N_{1}}{N_{2}}}
$$

$N_{1}$ and $N_{2}$ being the numbers of cells of the grids 1 and 2, respectively. In the following, $N$ will be used to indicate the total number of cells of a grid level, while $(1 / N)^{-1 / 3}$ is a parameter that represents adequately the grid resolution.

The difference between the values $f_{1}$ and $f_{h}=0$ is one of the error estimators. The actual fractional error is defined as:

$$
A_{1}=\frac{f_{1}-f_{h=0}}{f_{h=0}}
$$

Another error estimator, the relative error, is based on the difference between $f_{1}$ and $f_{2}$ :

$$
\varepsilon=\frac{f_{2}-f_{1}}{f_{1}}
$$

This quantity has to be corrected to take into account $r$ and $p$. The estimated fractional error for $f_{1}$ is therefore defined as:

$$
E_{1}=\frac{\varepsilon}{r^{p}-1}
$$

Although $E_{1}$ is based on a rational theory, it is not a bound on the error. On the contrary the Grid Convergence Index (GCI) provides an error band, i.e. a tolerance on the accuracy of the solution (Roache, 1998). The GCI on the fine grid is then defined as:

$$
G C I_{\text {fine }}=\frac{F_{S}|\varepsilon|}{\left(r^{p}-1\right)}
$$

where $F_{\mathrm{S}}$ is a safety factor, that is recommended to be 3.0 when comparing the results of two grids, and 1.25 for comparison of three grids (being this latter our case). The above defined error estimators have been all calculated, and are reported in Tab. 4 for $\mathrm{Q}^{*}$ and $\mathrm{P}^{*}$.

The values of heat flux $\left(\mathrm{Q}^{*}\right)$ and pressure $\left(\mathrm{P}^{*}\right)$ are reported in Fig. 22 for the three grid levels in function of the grid resolution (i.e. the parameter $(1 / N)^{-1 / 3}$ ) and compared with the value corresponding to zero grid spacing (computed by means of the Richardson extrapolation). 


\begin{tabular}{|c|c|c|}
\hline Error Indices & $\mathbf{Q}^{*}\left(\mathbf{W} / \mathbf{m}^{2}\right)$ & $\mathbf{P}^{*}(\mathrm{~Pa})$ \\
\hline$e p s$ & 0.0402 & 0.0097 \\
$E 1$ & 0.0134 & 0.0032 \\
GCI & $\mathbf{0 . 0 1 6 7}$ & $\mathbf{0 . 0 0 4 0}$ \\
$A 1$ & -0.0132 & -0.0032 \\
\hline
\end{tabular}

Table 4. Grid error indices

These plots confirm the right trend of solution grid convergence both for heat flux and pressure. In fact, the difference existing between the results of the coarse grid level and the medium one decreases if comparing the medium level with the fine one, and the trend of solution is towards the Richardson extrapolated value.

As a consequence, the Grid Convergence Index provides a level of confidence of the solution, therefore it can be concluded that (see Tab. 4):

- the error committed on the heat flux value with the finer grid level should be lower than $1.67 \%$;

- the error committed on the pressure value with the finer grid level should be lower than $0.40 \%$.

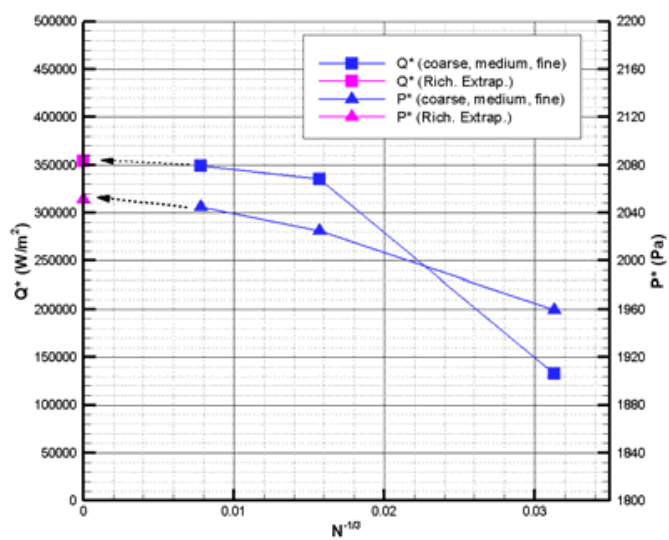

Fig. 22. Grid convergence estimation for heat flux $\left(\mathrm{Q}^{*}\right)$ and pressure $\left(\mathrm{P}^{*}\right)$ at the selected points

\subsection{Estimation of uncertainties}

An assessment of the uncertainty level related to test requirements fulfilment in terms of heat flux and pressure to be realized over the test-article is provided in this subsection, both for test design and test execution phases (Rufolo et al., 2008). The high complexity of involved phenomena together with the heterogeneous character of the different error sources make it impossible to give a rigorous definition and quantification of the error, but only a simplified estimation can be pursued.

Fig. 23 reports the entire process of numerical test design and test execution: during the design phase, starting from test requirements, a CFD aided activity is carried out in order to derive the proper settings for the heat flux $\left(\mathrm{Q}_{\mathrm{s}}\right)$ and pressure $\left(\mathrm{P}_{\mathrm{s}}\right)$ over the PWT calibration probe; in the testing phase the facility driving parameters (mass flow and arc current) are tuned in order to get the desired couple $\left(\mathrm{Q}_{\mathrm{s}}, \mathrm{P}_{\mathrm{s}}\right)$ over the calibration probe, then the test is executed and with 


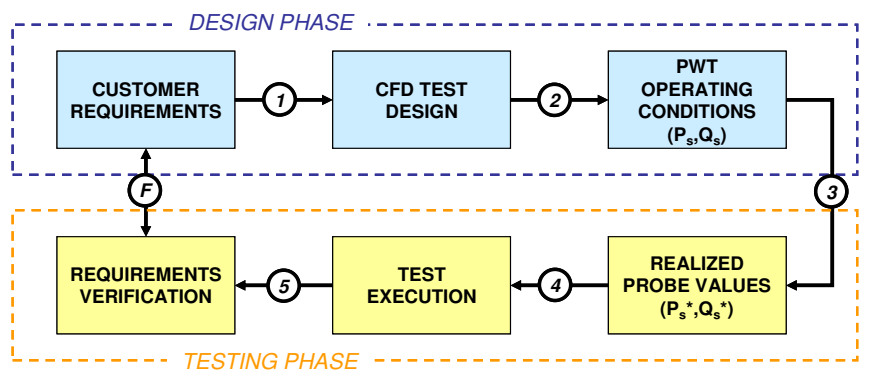

Fig. 23. Numerical test design and test execution chain

the post-test analysis it is finally possible to verify the matching of the requirements. Obviously, an error $\varepsilon_{i}$ is linked to each phase of the above described chain, and all of them contribute in determining the difference between the original requirements and their actual realization. It has to be said that in the present case the requirements were expressed in terms of heat flux and pressure for a fully catalytic and isothermal cold wall, and this is clearly a not realistic hypothesis for the kind of material and type of test to be conducted.

Moreover, during the test no heat flux direct measurements have been provided, and only an indirect derivation from temperature measurements can be obtained assuming radiative equilibrium at the wall (i.e. neglecting conduction into the material). In order to fully exploit measurements it is needed to associate correct values of catalytic recombination and emissivity coefficients, but these data have not been available during the project.

For these reasons, being unfeasible to characterize the complete error chain, only the following components of the error chain will be described hereinafter (Rufolo et al., 2008):

- how the test requirement is translated by means of CFD into PWT conditions ( $\varepsilon_{2}$ in Fig. 23);

- how the error in the experimental realization of the set point propagates on the requirements over the test-article ( $\varepsilon_{3}$ in Fig. 23).

The evaluation of the error $\varepsilon_{3}$ propagation is made by substituting the facility with its numerical modelling.

The numerical setting of PWT operating conditions comes out from an iterative process in which the facility driving conditions $\left(\mathrm{H}_{0}, \mathrm{P}_{0}\right)$ are tuned in order to match the requirements in terms of heat flux and pressure over the model to be tested (Di Benedetto et al., 2007). The error related to this process is definitively negligible, in the sense that it is always possible to find a couple $\left(\mathrm{H}_{0}, \mathrm{P}_{0}\right)$ that allows to numerically satisfy the requirements whichever is the accuracy prescribed. At the end of this process, when the correct couple $\left(\mathrm{H}_{0}, \mathrm{P}_{0}\right)$ has been found, the simulation of the flow field around the calibration probe is carried out in order to find out the couple $\left(Q_{s}, P_{s}\right)$ that will be used for the test execution (Di Benedetto et al., 2007). The process that translates the reservoir condition $\left(\mathrm{H}_{0}, \mathrm{P}_{0}\right)$ in local parameters $\left(\mathrm{Q}_{\mathrm{s}}, \mathrm{P}_{\mathrm{s}}\right)$ by means of a numerical modelling is affected by an error, above defined as $\varepsilon_{2}$.

By following the classical taxonomy adopted for CFD (AIAA, 1998) it is possible to recognize the following three error components for $\varepsilon_{2}$ :

- the Modelling Error (Chemical processes, fluid properties, Initial and Boundary conditions, Geometry representation, Turbulence Model);

- $\quad$ the Discretization Error (Grid independence, algorithm error);

- the Iteration Error (Convergence criterion). 
The modelling error is by far the most complex source of uncertainty to estimate. The common practice (AIAA, 1998) relies on the validation of the numerical code with respect to experimental measurements obtained for simple test cases. Unfortunately, the experimental measurement it is affected by an error that, especially in the case of heat flux measurements for aerothermodynamic tests, can make void the validation process.

As reported in (Ranuzzi \& Borreca, 2006) a series of comparisons with existing literature experiments were carried out during the development and validation phase of the H3NS CFD code. In particular, it was decided to refer to the Hyperboloid Flare Test Case carried out at the F4 blow-down arc heated high enthalpy facility of the ONERA in order to find out an error level applicable to the present case (Rufolo et al., 2008). The freestream Mach number is 8.7 , the total enthalpy is about $13 \mathrm{MJ} / \mathrm{Kg}$, the wall is considered isothermal at a temperature of $300 \mathrm{~K}$ and fully catalytic. Trying to find out an estimation of the modelling error related to the phenomenon we are interested in (heat flux and pressure along the testarticle flat panel), it is possible to extract the average percentage error for the measurement stations located in the mid part of the hyperboloid and ahead of the flare. In this way an error of about $4 \%$ for heat flux and $3 \%$ for pressure is obtained.

Another possibility for estimating the modelling error, in absence of affordable experimental results, is to carry out a sensitivity analysis with respect, for instance, to chemical model and/or transport properties model. With respect to the transport properties model, results obtained for the hyperboloid flare show no significant effect on pressure, while for heat flux the maximum deviation is about $3.1 \%$. As for the chemical models, a dedicated analysis has been carried out both for the PWT calibration probe and for the SPS test-article. The four different chemical models implemented in H3NS (Ranuzzi \& Borreca, 2006) have been tested: Kang-Dunn (Dunn \& Kang, 1997), Park 1990 (Park, 1990), Park-Rakich (Rakich et al., 1983) and Park 1993 (Park \& Lee, 1993), this latter being the chemical model used for all the simulations performed in the present activity. Regarding the stagnation point of the calibration probe, the largest deviation occurs for the Kang-Dunn model (2.63\% for heat flux and $0.97 \%$ for pressure). For what concerns the SPS test-article simulation, the percentage deviations of heat flux at the beginning of the flat panel and of maximum pressure over the flat panel obtained with Kang-Dunn model with respect to the Park 1993 results are respectively $0.38 \%$ and $3.13 \%$.

For what concerns the discretization error, the results of the grid convergence analysis of the three-dimensional simulation of the FLPP-SPS test-article, reported in Section 5.2, show that, with respect to an ideal zero-spacing grid, an error of $1.67 \%$ on the heat flux at the beginning of the flat panel and of $0.40 \%$ on the maximum pressure on the panel is committed.

For what concerns the iteration error, it has to be said that, even if we are interested in achieving the steady state solution of the Navier-Stokes equations, when the flow field to be resolved contains features characterized by intrinsic unsteadiness (e.g. recirculation bubble, vortex shedding, shock wave instability), the residue of the equations does not decrease towards the machine precision. Despite the presence of these unsteadiness, the quantities of interest in our case, as the heat flux and the pressure over the flat panel, reached a steady state value so that the iteration error can be neglected.

Trying to summarize, Tab. 5 reports the identified uncertainties (intended as estimation of the errors). The last column of the table reports the "overall error" obtained adding all the components.

Concerning the error $\varepsilon_{3}$, it is needed to estimate how the experimental uncertainty on the measurements of heat flux and pressure over the calibration probe translates in uncertainty 


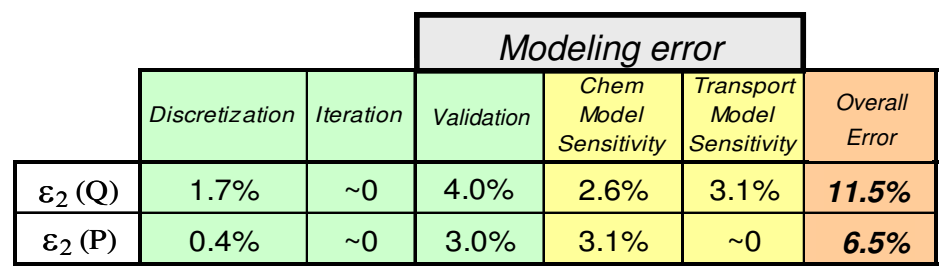

Table 5. Summary of identified error components

of the requirements over the test article. This can be done only by adopting the CFD tool as transfer function. The error associated to the heat flux measurement of the calibration probe is $\pm 90 \mathrm{~kW} / \mathrm{m}^{2}$, while the one associated to the stagnation pressure measurement is \pm 1.1 mbar (Marini et al., 2002). Starting from the values realized during the test, four couples $\left(Q_{s}\right.$, $\mathrm{P}_{\mathrm{s}}$ ) have been identified by adding and subtracting their own error both to Qs and Ps; the corresponding values are reported in the first two columns of Tab. 6. For each couple, the facility driving conditions $\left(\mathrm{H}_{0}, \mathrm{P}_{0}\right)$ have been found by following the iterative process already described (Di Benedetto et al., 2007) (columns three and four of Tab. 6), and then two-dimensional simulations of both probe and model (i.e. test article) have been carried out for each condition. The percentage errors referred to the nominal values are reported in Tab. 6 for each of the four conditions.

\begin{tabular}{|c|c|c|c|c|c|c|c|c|c|c|c|c|}
\hline & \multicolumn{4}{|c|}{ PROBE EXP } & \multirow{2}{*}{\multicolumn{4}{|c|}{ PROBE CFD }} & \multicolumn{4}{|c|}{ MODEL CFD } \\
\hline & & & & & & & & & & IT \#4 & & $\Gamma \# 1$ \\
\hline & $\begin{array}{c}\text { Ps } \\
\text { [mbar] }\end{array}$ & $\begin{array}{c}\text { Qs } \\
{[\mathrm{kW} / \mathrm{m} 2]}\end{array}$ & $\begin{array}{c}\text { P0 } \\
\text { [bar] }\end{array}$ & $\begin{array}{c}\mathrm{HO} \\
{[\mathrm{MJ} / \mathrm{kg}]}\end{array}$ & $\begin{array}{c}\text { Ps } \\
\text { [mbar] }\end{array}$ & err\% & $\begin{array}{c}\text { Qs } \\
{[\mathrm{kW} / \mathrm{m} 2]}\end{array}$ & err\% & $\begin{array}{c}\mathrm{Ps} \\
\text { [mbar] }\end{array}$ & err\% & $\begin{array}{c}\text { Qs } \\
{[\mathrm{kW} / \mathrm{m} 2]}\end{array}$ & err\% \\
\hline$\left(p_{\text {req }}, q_{\text {req }}\right)$ & 34.2594 & 2121.82 & 4.90 & 17.40 & 34.26 & & 2121.82 & & 23.84 & & 338.66 & \\
\hline$\left[p_{\text {req }}, q_{\text {req }}+\operatorname{err}(q)\right]$ & 34.2594 & 2211.82 & 4.88 & 18.03 & 34.25 & $0.02 \%$ & 2211.56 & $4.23 \%$ & 23.82 & $0.08 \%$ & 353.02 & $4.24 \%$ \\
\hline$\left[p_{\text {req }}, q_{\text {req }}-\operatorname{err}(q)\right]$ & 34.2594 & 2031.82 & 4.93 & 16.81 & 34.26 & $0.01 \%$ & 2030.47 & $4.31 \%$ & 23.84 & $0.02 \%$ & 325.32 & $3.94 \%$ \\
\hline$\left[p_{\text {req }}+\operatorname{err}(p), q_{\text {req }}\right]$ & 35.3594 & 2121.82 & 5.07 & 17.18 & 35.35 & $3.17 \%$ & 2122.05 & $0.01 \%$ & 24.58 & $3.12 \%$ & 339.47 & $0.24 \%$ \\
\hline$\left[p_{\text {req }}-\operatorname{err}(p), q_{\text {req }}\right]$ & 33.1594 & 2121.82 & 4.73 & 17.64 & 33.18 & $3.14 \%$ & 2125.11 & $0.16 \%$ & 23.09 & $3.14 \%$ & 338.39 & $-0.08 \%$ \\
\hline
\end{tabular}

Table 6. Influence of calibration probe measurements uncertainty on test article requirements

Regarding the model, the errors were evaluated with respect to the beginning of the flat panel for the heat flux (Point \#1 in Tab. 6) and to the maximum value over the flat panel for the pressure (Point \#4 in Tab. 6). It can be seen that in the worst case the difference between the errors on the probe and on the model are limited to $0.37 \%$ for the heat flux and $0.05 \%$ for the pressure. So it can be stated that, within the approximation related with the numerical process, the experimental uncertainties on the point settings is identically transferred to test article requirements.

In conclusion, the analysis reported above has been aimed at deriving an estimation of the errors $\varepsilon_{2}$ and $\varepsilon_{3}$. Obviously, the analysis cannot be considered exhaustive and especially for the CFD related error only a very simplified indication has been provided. As a matter of fact, the two errors $\varepsilon_{2}, \varepsilon_{3}$ can be considerer fully independent.

At worst, for the present case the estimated overall errors are about $15 \%$ on heat flux and $9.5 \%$ on pressure.

\section{Rebuilding CFD activity}

The FLPP-SPS TPS demonstrator plasma wind tunnel test was successfully performed on September 20th, 2007 simulating a 15 min re-entry trajectory in three steps characterized by 
increasing total enthalpy level in test chamber, i.e. increasing continuously wall heat flux (Trifoni et al., 2007).

The test condition, which the CFD three-dimensional analysis described in the previous section refers to, corresponds to the second test step, defined as the "nominal" one. This latter condition has been rebuilt after the test by exploiting the calibration probe heat flux and pressure available measurements.

A different hypothesis about the temperature wall condition has been made, in order to simulate a more realistic condition with respect to the hypothesis of cold wall of the pre-test CFD simulation. In particular, radiative wall temperature has been computed assuming the equilibrium between the convective and the radiative heat fluxes. The emissivity coefficient has been provided by SPS $(\varepsilon=0.8)$, while the hypothesis of fully catalytic surface has been maintained also in the test rebuilding CFD simulation, as also indicated by SPS.

In Fig. 24 the CAD model (left) is compared with the model as built (right), in which there is no step in the bottom part. However, this difference in the test article configuration should involve discrepancies only on the regions closer to the bottom part of the model, therefore no influence is expected on the flat and curved panels.
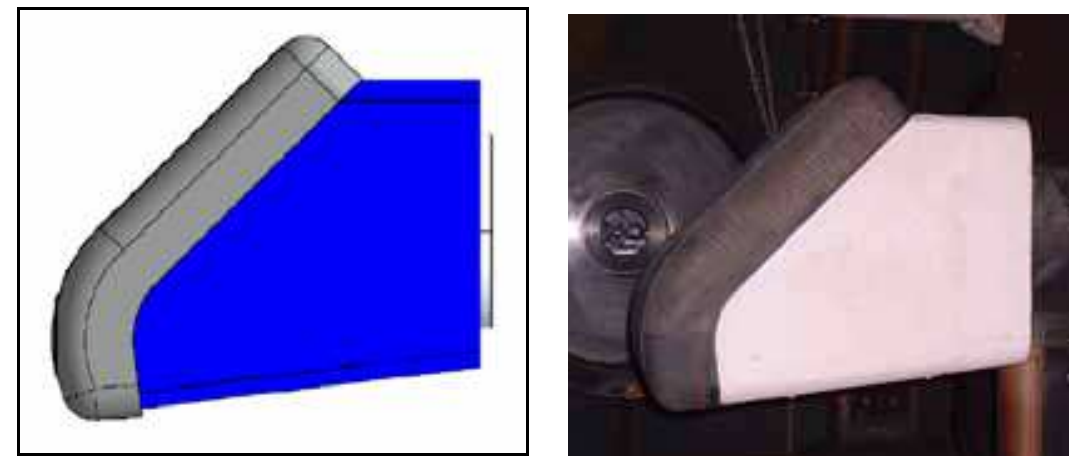

Fig. 24. CAD model (left) and model as built (right)

\subsection{Operating condition assessment}

The pre-test three-dimensional CFD simulation has been carried out in the PWT operating condition resulting from the previous CFD test design activity (Rufolo et al., 2008), whose results are reported in Tab. 7 .

\begin{tabular}{|c|c|c|}
\hline Design Test Chamber & $\mathbf{P}_{\mathbf{0}}\left(\mathbf{b a r}_{\mathbf{a}}\right)$ & $\mathbf{H}_{\mathbf{0}}(\mathbf{M} \mathbf{J k g})$ \\
\cline { 2 - 3 } Conditions & 5.20 & 16.70 \\
\hline $\begin{array}{c}\text { Calibration Probe } \\
\text { Stagnation Point (CFD) }\end{array}$ & $\mathbf{P}_{\mathbf{S}}\left(\mathbf{m b a r}_{\mathbf{a}}\right)$ & $\mathbf{Q} \mathbf{s}\left(\mathbf{k W} / \mathbf{m}^{\mathbf{2}}\right)$ \\
\cline { 2 - 3 } & 36.15 & 2070 \\
\hline
\end{tabular}

Table 7. PWT test design operating condition

This condition has been compared, in terms of heat flux and pressure on the PWT hemispherical calibration probe, with that actually measured during the second step (the "nominal" one) of the test. These latter values are reported in Tab. 8, together with their error bars (Trifoni et al., 2007). 
In order to reproduce in the rebuilding CFD simulation the same condition realized in test chamber during the test in terms of total pressure and total enthalpy, the iterative procedure described in (Rufolo et al., 2008) and (Di Benedetto et al., 2007) has been applied, this time having as requirements the values measured on the calibration probe.

\begin{tabular}{|c|c|c|}
\hline $\begin{array}{c}\text { Calibration Probe } \\
\text { Stagnation Point } \\
\text { (Measured) }\end{array}$ & $\mathbf{P}_{\mathbf{S}}\left(\mathbf{m b a r}_{\mathbf{a}}\right)$ & $\left.\mathbf{Q}_{\mathbf{S}} \mathbf{( k W} / \mathbf{m}^{\mathbf{2}}\right)$ \\
\cline { 2 - 3 } & $34.20 \pm 1.1$ & $2120 \pm 90$ \\
\hline
\end{tabular}

Table 8. Values at the calibration probe stagnation point measured during the test

Finally, the PWT operating condition obtained for the rebuilding CFD activity is summarized in Tab. 9.

\begin{tabular}{|c|c|c|}
\hline $\begin{array}{c}\text { Rebuilding Test } \\
\text { Chamber Conditions }\end{array}$ & $\mathbf{P}_{\mathbf{0}}\left(\mathbf{b a r}_{\mathbf{a}}\right)$ & $\mathbf{H}_{\mathbf{0}}$ (MJlkg) \\
\cline { 2 - 3 } & 4.90 & 17.40 \\
\hline $\begin{array}{c}\text { Calibration Probe } \\
\text { Stagnation Point (CFD) }\end{array}$ & $\mathbf{P}_{\mathbf{S}}(\mathbf{m b a r})$ & $\mathbf{Q s}_{\mathbf{s}}\left(\mathbf{k W} / \mathbf{m}^{\mathbf{2}}\right)$ \\
\cline { 2 - 3 } & 34.25 & 2121 \\
\hline
\end{tabular}

Table 9. PWT test rebuilding operating condition

\subsection{Three-dimensional results}

The three-dimensional CFD rebuilding simulation has been performed in the PWT "nominal" test condition of Tab. 9. The more realistic radiative equilibrium wall condition, with surface emissivity $\varepsilon=0.8$, has been imposed instead of the cold wall. In order to qualitatively evaluate the actual catalysis of the CMC panels through comparison with temperature measurements, both fully catalytic (FC) and non catalytic (NC) wall conditions have been considered.

Heat flux distribution together with the skin-friction lines pattern on the test article is shown in Fig. 25: heat flux on the stagnation line is about $600 \mathrm{~kW} / \mathrm{m}^{2}$ for FC case, and it decreases to $200 \mathrm{~kW} / \mathrm{m}^{2}$ for NC one. Temperature contour maps are shown in Fig. 26: in the FC case the local maximum values of temperature are around $2000 \mathrm{~K}$ on the stagnation line and about $2200 \mathrm{~K}$ on the roundings of lateral fairings of the curved panel. On the flat panels the predicted temperature ranges from about $1500 \mathrm{~K}$ (in the single panel central area) to about $1800 \mathrm{~K}$ at the panel lateral edges. Temperature levels of about $1000 \mathrm{~K}$ are predicted on the lateral sides of the test article. These values are quite strongly reduced with the NC assumption (about $500 \mathrm{~K}$ on the stagnation line), due to a combined effect of the high energy content of the flow and the large bluntness of the test article.

The analysis which follows refers to FC condition results only, this in order to make possible a comparison with the pre-test numerical findings. An enlargement of the model top frame with skin-friction lines coloured by shear stress value is reported in Fig. 27 (left) and compared with the distribution obtained in the pre-test simulation (right). The phenomenology and the shear stress distribution are very similar to those predicted in the pre-test activity, while a slightly larger separated area is observed as a consequence of the changed wall temperature condition. In fact, a higher surface temperature implies a boundary layer thickening (in particular of the subsonic region), in this way increasing the upstream and downstream pressure disturbance propagation. As a consequence of the 

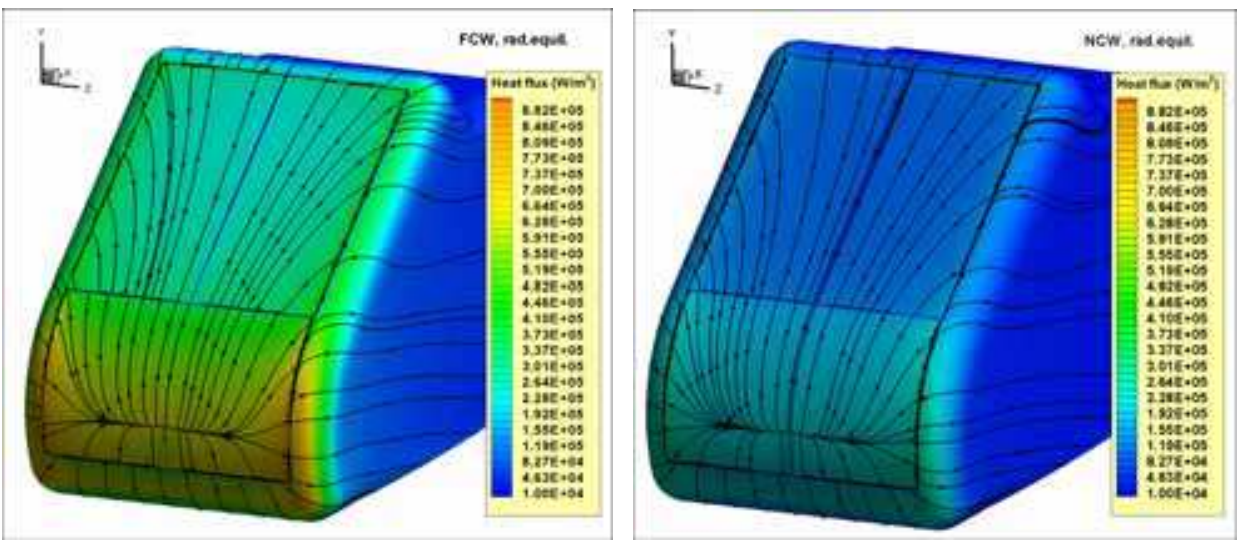

Fig. 25. Heat Flux contour map with skin-friction lines; FC (left), NC (right)
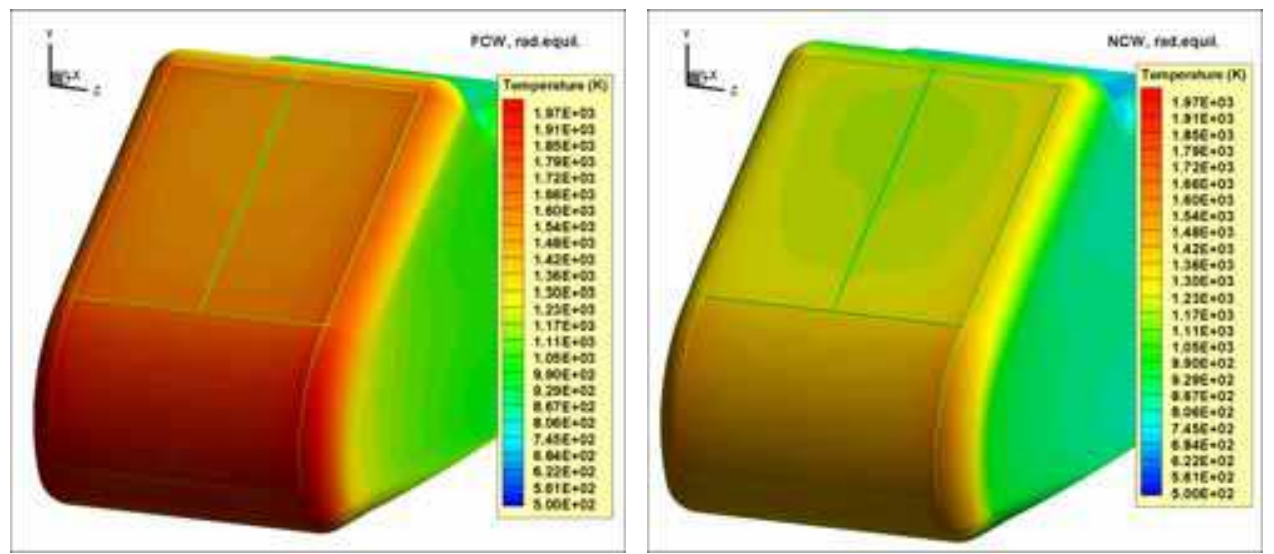

Fig. 26. Temperature contour map; FC (left), NC (right)
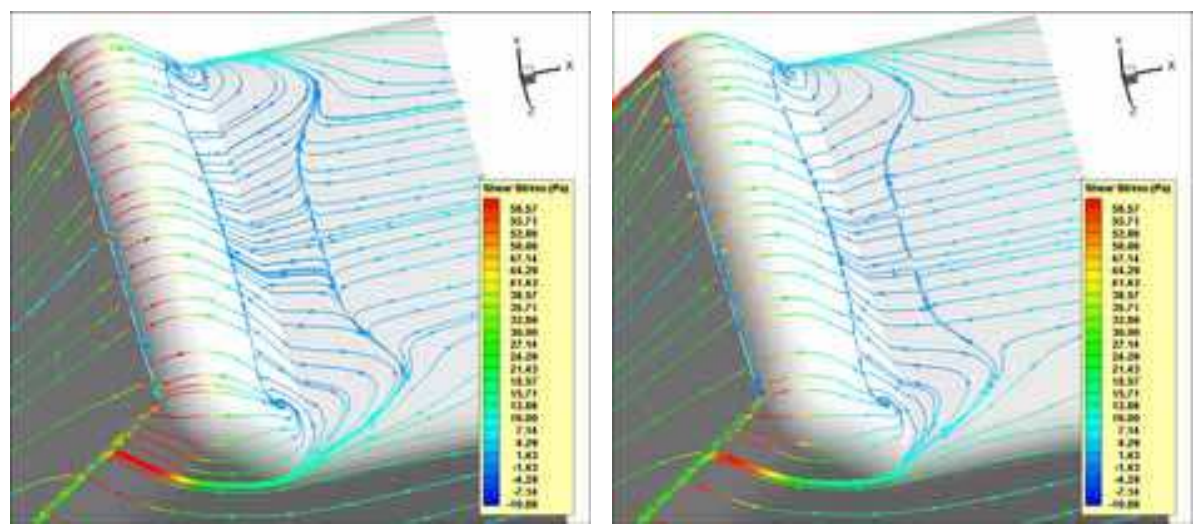

Fig. 27. Enlargement of the model top frame; skin-friction coloured by the shear stress; rebuilding (radiative equilibrium, left), pre-test (cold wall, right) 

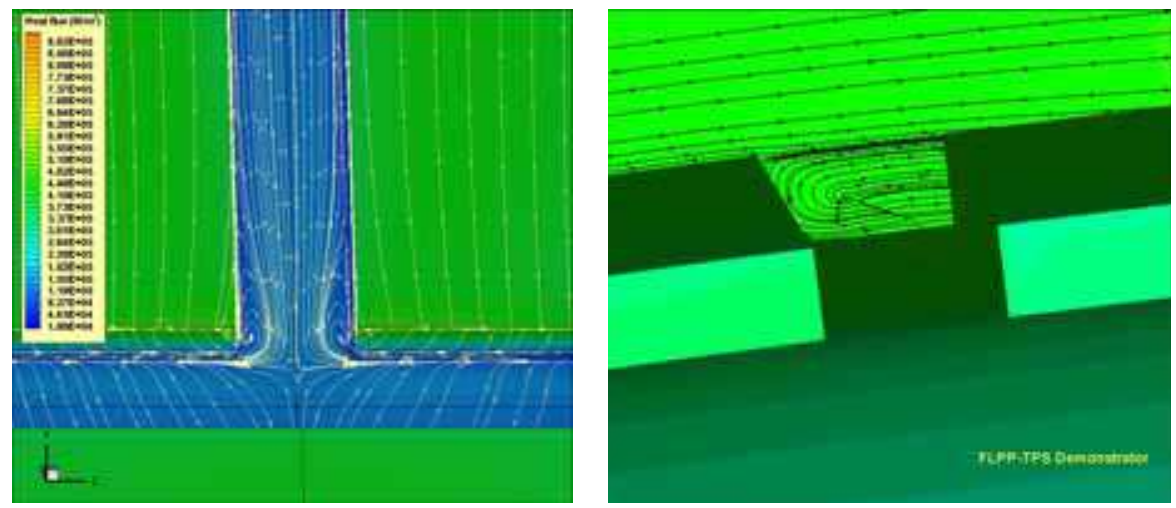

Fig. 28. T-gap heat flux contour map with skin-friction lines (left) and longitudinal gap recirculation (right)
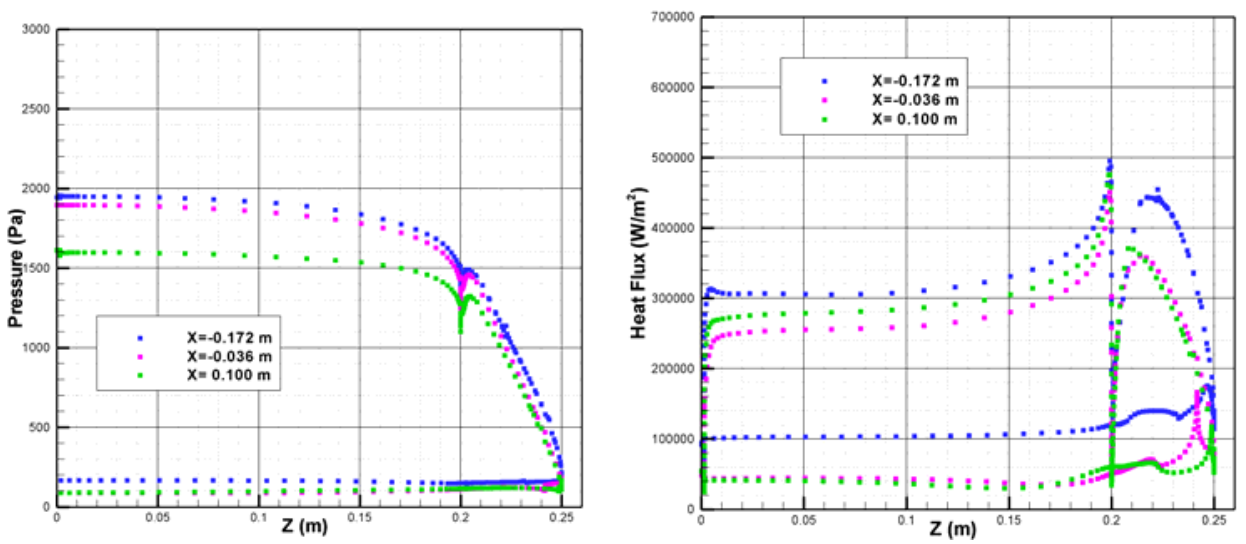

Fig. 29. Transversal pressure (left) and heat flux (right) distributions
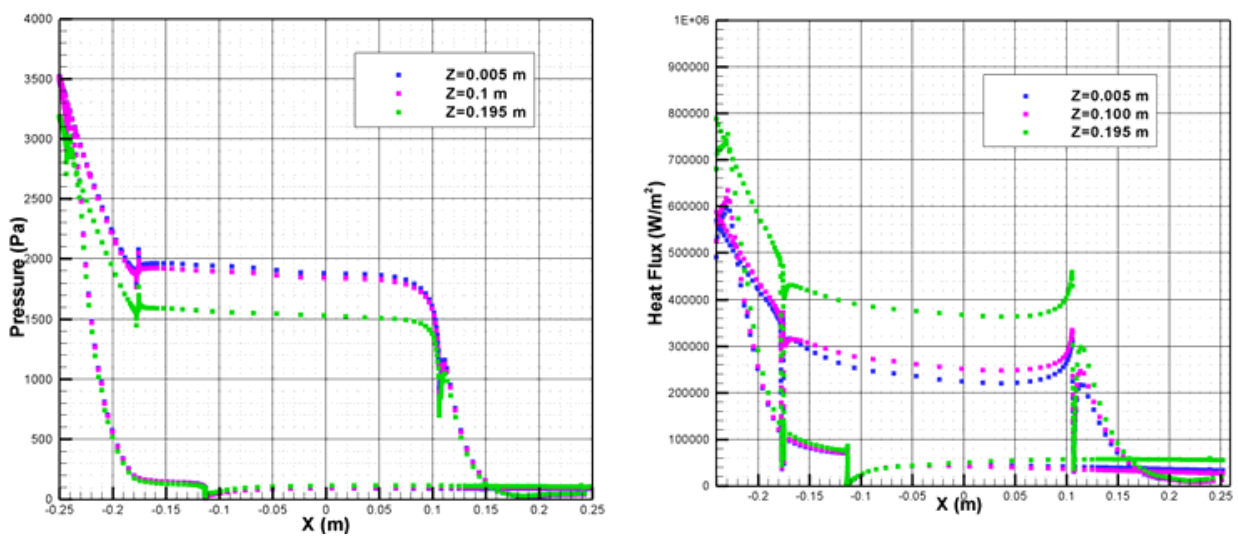

Fig. 30. Longitudinal pressure (left) and heat flux (right) distributions 
increased temperature, an extension of the regions submitted to higher shear stress is observed, although the overall structure of the flow seems unchanged.

The flow inside the T-gap is described in Fig. 28. The interaction between the transversal stream and the longitudinal one realizes in a saddle point and in two lateral vortices, but with a different flow pattern with respect to the pre-test simulation due to the effects of the surface temperature wall condition (see Fig. 14 and Fig. 15). The vortex flow inside the transversal gap is again characterized by a strong spanwise velocity component that increases moving towards the edge, a inner vortex at the base of the panel and an attachment line at the front edge of the panel. As expected, the region of high heat flux at the front edge of the flat panel, and in particular at the top corner, is largely reduced.

Pressure and heat flux distributions in transversal and longitudinal directions are shown, respectively, in Fig. 29 and Fig. 30. The main flow features, already described in Section 5.1 (see from Fig. 18 to Fig. 21), are all confirmed by the present test CFD rebuilding, although quantitative levels are different due either to the realization of a slightly different "nominal" condition, with respect to that analyzed during the pre-test CFD activity, either to the different surface thermal boundary condition.

At the flat panel leading edge, CFD rebuilding simulation yields a heat flux of about 440 $\mathrm{kW} / \mathrm{m}^{2} 5 \mathrm{~mm}$ from the lateral edge $(\mathrm{Z}=0.195 \mathrm{~m})$, and it is slightly larger than $300 \mathrm{~kW} / \mathrm{m}^{2}$ for the rest of the panel (Fig. 29-right). Downstream along the panel heat flux remains around $300 \mathrm{~kW} / \mathrm{m}^{2}$ apart from the lateral edge, affected by the presence of the step, where 400 $\mathrm{kW} / \mathrm{m}^{2}$ all along the panel are predicted (Fig. 30-right).

Transversal and longitudinal pressure distributions over the model are reported in Fig. 29left and Fig. 30-left respectively; pressure is not significantly affected by spanwise effects, apart from the more lateral section $\mathrm{Z}=0.195 \mathrm{~m}$ where a strong flow expansion occurs: transversal distributions remain two-dimensional for most of the half panel span, as well as the longitudinal ones are flat enough for $80 \%$ of the panel length.

\section{CFD/Experiments comparison}

In this section some of the experimental data collected during the FLPP-SPS demonstrator test in the SCIROCCO PWT (Trifoni et al., 2007) are compared to the results of the numerical rebuilding described in Section 6.

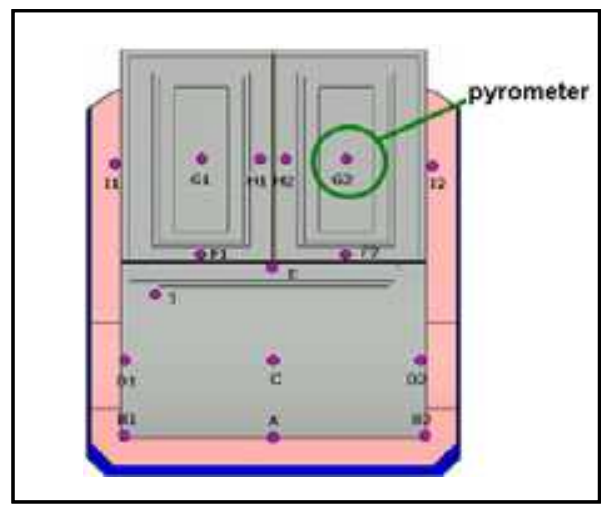

Fig. 31. Test article instrumentation 
During the test, eleven B-type thermocouples have measured the back wall temperatures of the CMC panels. Among these, those located on the flat panels which have correctly worked (F2-1, G2-1, H2-1, H1-1, see Fig. 31) have been selected to perform comparisons with CFD temperature distributions. Moreover, a dual colour pyrometer (range: 1000-3000 ${ }^{\circ} \mathrm{C}$ ) has been pointed to G2- 1 thermocouple location and two IR thermo-cameras ( $\varepsilon=0.8$, range: 600 $2500^{\circ} \mathrm{C}$ ) have been used to monitor the test article during the test both from the top (flat panels) and from the lateral front (curved panel area).

In Fig. 32 temperature measured by thermocouples is compared with CFD distributions along the two sections, indicated as slices in the figure, where thermocouples are located.

As expected, measured temperatures lie more or less in the middle between the non catalytic (NC) and the fully catalytic (FC) distributions. In addition, it has to be said that the surface temperatures can be estimated to be about $50^{\circ} \mathrm{C}$ higher than the measured back wall ones.

In Fig. 33, the same kind of comparison is reported for the temperature measured by the dual colour pyrometer. A lower emissivity value of 0.68 , which is a combination of the real emissivity value of the material and all the experimental uncertainty factors, allows to match pyrometer and thermal camera readings, as reported in Tab. 10 (experimental emissivity evaluation). Therefore, also the CFD temperatures in Fig. 33 have been properly scaled (to the emissivity value of $\varepsilon_{\exp }=0.68$ ) in the post-processing phase, in such a way to make the comparison meaningful and to reproduce as much as possible the actual wall conditions.

An attempt to derive an estimation of the CMC panels catalytic recombination coefficient has been done by combining the experimental results to a CFD-based correlation. Namely, by means of CFD two-dimensional computations with finite rate catalysis values at the wall, a function that relates the heat flux at a certain point of the flat panel with the recombination

\begin{tabular}{|c|c|c|}
\hline $\begin{array}{c}\mathrm{T} \\
\text { pyrometer }\end{array}$ & $\begin{array}{c}\mathrm{T} \\
\text { thermocamera }\end{array}$ & $\varepsilon_{\exp }$ \\
\hline $1500 \mathrm{~K}$ & $1360 \mathrm{k}$ & 0.68 \\
\hline
\end{tabular}

Table 10. Experimental emissivity evaluation

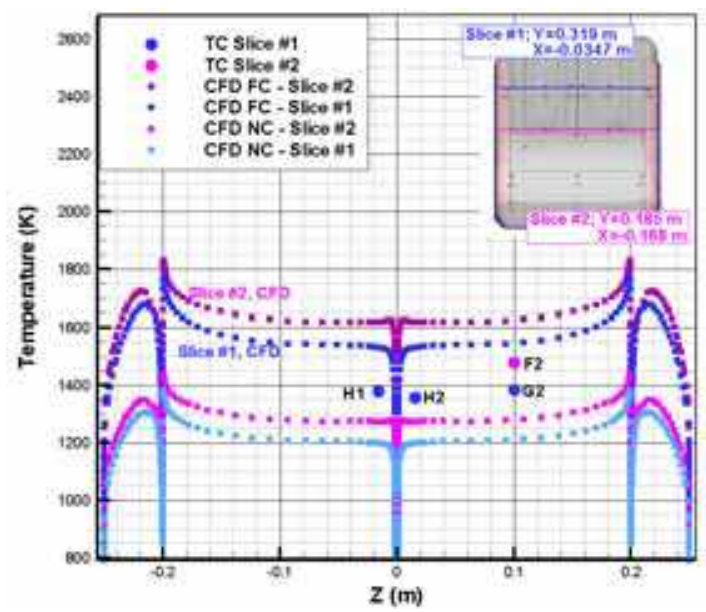

Fig. 32. Comparison between temperature CFD distributions and thermocouples measurements 


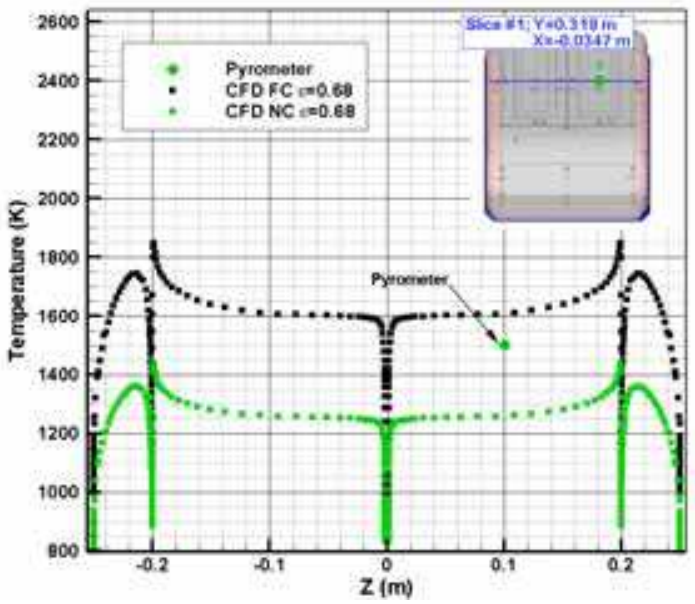

Fig. 33. Comparison between temperature CFD distributions and pyrometer measurement coefficient $\gamma$ has been derived. By crossing this function with the radiative heat flux corresponding to the pyrometer reading, a value for $\gamma$ of about 0.008 has been obtained. It has to be remarked that this value only represents a rough estimation and it includes all the numerical and experimental errors.

Finally, some qualitative comparisons of the bow shock wave shape are shown from Fig. 34 to Fig. 36, where the predicted flow field in the shock layer region has been overlapped to the images taken by the two video cameras during the test. In Fig. 34 and Fig. 35, the shock section extracted from CFD computation and the predicted temperature field in the shock region have been superimposed on a view from the top camera. The comparison shows that both shock shape and stand off distance predicted in the stagnation region well reproduce the actual ones. In Fig. 36 the predicted atomic nitrogen mass fraction is overlapped to a view from the side camera, showing a good agreement of predicted and actual shock shape around the entire model, and a significant presence of atomic nitrogen $(\mathrm{N})$ around most of the curved panel.

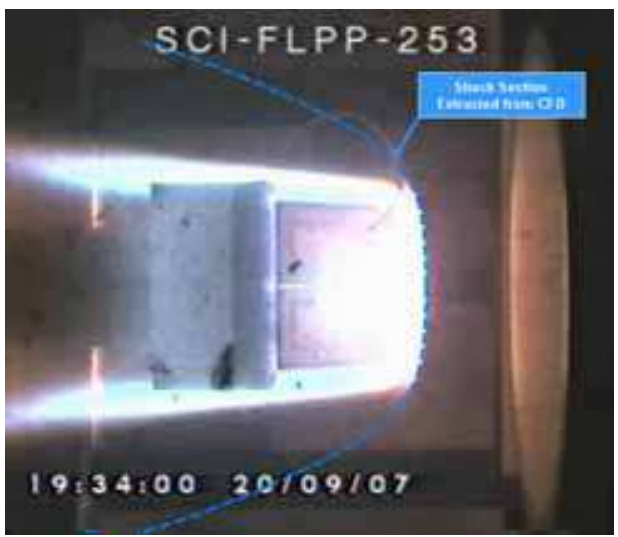

Fig. 34. Top view of the model during test. Comparison of predicted and actual shock shape 


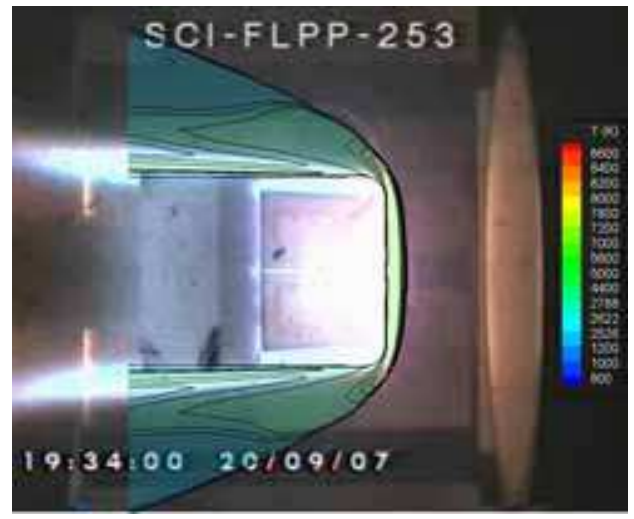

Fig. 35. Top view of the model during test. Comparison with predicted temperature contours

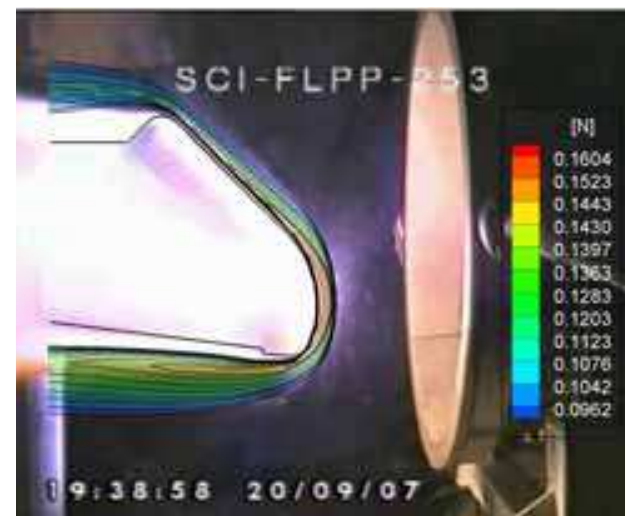

Fig. 36. Side view of the model during test. Comparison with predicted nitrogen concentration

\section{Conclusions}

This chapter has described the three-dimensional CFD activities carried out to support the SCIROCCO plasma wind tunnel test performed on the FLPP-SPS TPS demonstrator designed and manufactured by Snecma Propulsion Solide.

After a CFD pre-test activity, during which the test point previously designed by a simplified two-dimensional methodology has been verified and the final PWT test condition frozen, the post-test phase has regarded the plasma test CFD rebuilding.

The FLPP-SPS PWT test was performed with full success on September 20th, 2007 simulating a 15 minutes re-entry trajectory in three steps characterized by increasing total enthalpy levels in test chamber. The test condition which the present CFD three-dimensional analysis refers to corresponds to the second "nominal" step.

This latter condition has been rebuilt by exploiting the calibration probe heat flux and pressure available measurements, and by applying the same iterative procedure used 
during the test design phase, this time having as requirements the values measured on the calibration probe. Moreover, in order to perform more realistic simulations, radiative equilibrium has been imposed at the wall, whereas to qualitatively evaluate the actual CMC panels catalysis both FC and NC conditions have been considered.

Similar flow features have been predicted both in the pre-test phase and the post-test rebuilding phase, and some meaningful comparison between CFD rebuilding results and experimental findings have allowed to assess the full capability of the present CFD-based methodology to design and properly rebuild a plasma wind tunnel test, with its own accuracy bounds. In addition, an approach to determine the uncertainties related to both design and testing phases, with respect to the satisfaction of test requirements, has been presented.

Finally, a rough estimation of the catalyticity of the CMC panels under realistic re-entry conditions has been obtained by crossing experimental measurements and CFD results.

An important step for future applications like the present should be to rebuild plasma wind tunnel tests accounting for the actual catalytic behaviour of the different parts of the test article. Of course, to do this the proper experimental characterization of the involved materials in terms of recombination coefficients as functions of temperature and pressure is needed. Then, once having re-tuned the CFD methodology, the approach could be directly applied starting from the pre-test design phase.

\section{Acknowledgements}

This work has been fully supported by SPS in the frame of FLPP Materials \& Structures Technological Activities, Period 1, Phase 1, coordinated by NGL Consortium and supervised by the European Space Agency.

A special thank goes to the whole CIRA Plasma Wind Tunnel Team that made possible the FLPP-SPS test campaign.

\section{References}

Barreteau, R., Foucault, A., Parenteau, J.M., Pichon, T. (2008). Development and Test of a Large-Scale CMC TPS Demonstrator, 2nd International ARA DAYS, AA-3-2008-4, 2123 October, 2008, Arcachon, France.

Rufolo, G., Di Benedetto, S., Marini M. (2008) Theoretical-Numerical Design of a Plasma Wind Tunnel Test for a Large TPS Demonstrator, 6th European Symposium on Aerothermodynamics for Space Vehicles, paper s17_5, Versailles, France, November 2008.

Marini, M., De Filippis, F., Del Vecchio, A., Borrelli, S., Caristia S. (2002) CIRA 70-MW Plasma Wind Tunnel: A Comparison of Measured and Computed Exit Nozzle Flow Profiles, Euromech-440 Conference, 16-19 September 2002, France, Marseilles.

De Filippis, F. et al. (2003) The Scirocco PWT Facility Calibration Activities, 3rd International Symposium Atmospheric Reentry Vehicle and Systems, March 2003, Arcachon, France.

Ranuzzi, G., \& Borreca, S. (2006) CLAE Project. H3NS: Code Development and Validation, Internal Report CIRA-CF-06-1017, September 2006.

Di Clemente, M. (2008) Numerical studies for the realization of aerodynamic systems for guide and control of re-entry vehicles, Ph.D. Dissertation, Mechanics and Aeronautics Dept., La Sapienza Univ., Rome. 
Park, C. (1989) A Review of Reaction Rates in High Temperature Air, AIAA Paper 89-1740, June 1989.

Millikan, R.C., White, D.R. (1963) Systematic of Vibrational Relaxation, Journal of Chemical Physics, Vol. 39, No. 12, pp. 3209-3213.

Park, C., Lee, S. H. (1993) Validation of Multi-Temperature Nozzle Flow Code NOZNT, AIAA Paper 93-2862.

Yun, K. S., Mason, E. A. (1962) Collision Integrals for the Transport Properties of Dissociating Air at High Temperatures, Physics of Fluids, Vol. 5, No. 4, 1962, pp. 380-386.

Kee, R. J., Warnatz, J., Miller, J. A. (1983) A Fortran Computer Code Package for the Evaluation of Gas-Phase Viscosities, Conductivities and Diffusion Coefficients, Sandia Rept. SAND83-8209, March 1983.

Di Benedetto, S., Bruno, C. (2010) A Novel Semi-Empirical Model for Finite Rate Catalysis with Application to PM1000 Material, Journal of Thermophysics and Heat Transfer, Vol. 24, No. 1, January-March 2010, pp. 50-59.

Roache, P.J. (1998) Verification and Validation in Computational Science and Engineering, Hermosa Publishers, Albuquerque.

AIAA (1998) Guide for the Verification and Validation of Computational Fluid Dynamics Simulations, G-077-1998, January 14, 1998.

Dunn, M.G., Kang, S.W. (1973) Theoretical and experimental studies of reentry plasma. Technical Report NASA CR 2232, NASA.

Park, C. (1990) Nonequilibrium Hypersonic Aerothermodynamics, Wiley Interscience.

Rakich, J.V., Bailey, H.E., Park, C. (1983) Computation of nonequilibrium, supersonic threedimensional inviscid flow over blunt-nosed bodies, AIAA Journal Vol. 21, June 1983, pp. 834-841, ISSN 0001-1452.

Trifoni, E. et al. (2007) DD[3] - PWT Test Report, Internal Report CIRA-TR-07-0230, November 2007.

Di Benedetto, S., Di Clemente, M., Marini, M. (2007) Plasma Wind Tunnel Test Design Methodologies for Re-entry Vehicle Components, 2nd European Conference for Aerospace Sciences (EUCASS), paper N. 228, 1-6 July 2007, Brussels, Belgium. 


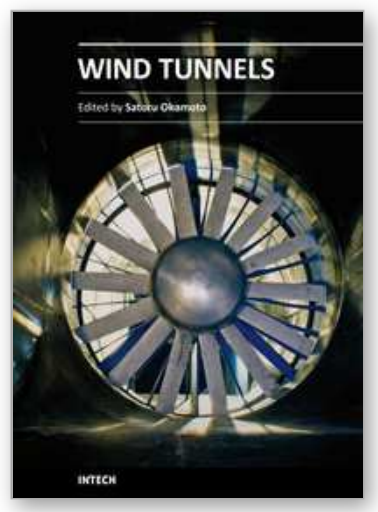

\author{
Wind Tunnels \\ Edited by Prof. Satoru Okamoto
}

ISBN 978-953-307-295-1

Hard cover, 136 pages

Publisher InTech

Published online 10, February, 2011

Published in print edition February, 2011

Although great advances in computational methods have been made in recent years, wind tunnel tests remain essential for obtaining the full range of data required to guide detailed design decisions for various practical engineering problems. This book collects original and innovative research studies on recent applications in wind tunnel tests, exhibiting various investigation directions and providing a birdâ€ $€^{\mathrm{TM}}$ s eye view on this broad subject area. It is composed of seven chapters that have been grouped in two major parts. The first part of the book (chapters 1â€"4) deals with wind tunnel technologies and devices. The second part (chapters 5â€"7) deals with the latest applications of wind tunnel testing. The text is addressed not only to researchers but also to professional engineers, engineering lecturers, and students seeking to gain better understanding of the current status of wind tunnels.ã€€Through its seven chapters, the reader will have an access to a wide range of works related to wind tunnel testing.

\title{
How to reference
}

In order to correctly reference this scholarly work, feel free to copy and paste the following:

Sara Di Benedetto, Giuseppe C. Rufolo, Marco Marini and Eduardo Trifoni (2011). Rebuilding and Analysis of a SCIROCCO PWT Test on a Large TPS Demonstrator, Wind Tunnels, Prof. Satoru Okamoto (Ed.), ISBN: 978-953-307-295-1, InTech, Available from: http://www.intechopen.com/books/wind-tunnels/rebuilding-andanalysis-of-a-scirocco-pwt-test-on-a-large-tps-demonstrator

\section{INTECH}

open science | open minds

\section{InTech Europe}

University Campus STeP Ri

Slavka Krautzeka 83/A

51000 Rijeka, Croatia

Phone: +385 (51) 770447

Fax: +385 (51) 686166

www.intechopen.com

\section{InTech China}

Unit 405, Office Block, Hotel Equatorial Shanghai

No.65, Yan An Road (West), Shanghai, 200040, China

中国上海市延安西路65号上海国际贵都大饭店办公楼 405 单元

Phone: +86-21-62489820

Fax: $+86-21-62489821$ 
(C) 2011 The Author(s). Licensee IntechOpen. This chapter is distributed under the terms of the Creative Commons Attribution-NonCommercialShareAlike-3.0 License, which permits use, distribution and reproduction for non-commercial purposes, provided the original is properly cited and derivative works building on this content are distributed under the same license. 